\title{
SOME REMARKS ON ALMOST PERIODIC FUNCTIONS IN VIEW OF THE LEBESGUE MEASURE WITH APPLI- CATIONS TO LINEAR DIFFERENTIAL EQUATIONS
}

\author{
Dariusz Bugajewski and Adam Nawrocki \\ Adam Mickiewicz University, Faculty of Mathematics and Computer Science \\ ul. Umultowska 87, 61-614 Poznań, Poland; ddbb@amu.edu.pl \\ Adam Mickiewicz University, Faculty of Mathematics and Computer Science \\ ul. Umultowska 87, 61-614 Poznań, Poland; adam.nawrocki@amu.edu.pl
}

\begin{abstract}
In this paper we are going to investigate some properties of almost periodic functions in view of the Lebesgue measure with particular emphasis on their behavior under convolution. These considerations allow us to establish the main result concerning almost periodic in view of the Lebesgue measure solutions to linear differential equations of the first order. We also apply the theory of continued fractions to examine asymptotic behavior of a certain classical almost periodic function of that type. For that purpose we provide a new general method of calculation of certain type of limits.
\end{abstract}

\section{Introduction}

The theory of almost periodic functions was started by Danish mathematician Bohr (see $[3,4,5]$ ) who in the years 1924-1926 published series of very extensive and careful lectures in which, basing on so-called relatively dense sets, he introduced, in particular, the precise notion of a continuous uniformly almost periodic function. Therefore uniformly almost periodic (briefly: u.a.p.) functions are commonly called Bohr almost periodic (briefly: B-a.p.) functions. Let us add that Bohr based his investigation on earlier considerations of Bohl and Escalangon. Almost periodic functions present natural generalization of periodic functions from the point of view of some algebraic properties as well as from a totally different point of view in the theory of function spaces.

There are many generalizations of B-a.p. functions. Among these generalizations a particular role seem to play almost automorphic functions (see e.g. [7, 8, 14] and many other papers on that topic). Another very important classes of almost periodic functions were introduced by Stepanov [17] in 1926, when he was dealing with locally integrable functions in view of some integrable metrics. Now, in the literature such functions are called Stepanov almost periodic (briefly: $S^{p}$-a.p.) functions. Let us add that for functions of such type, as in the case of u.a.p. functions, there exists the mean value and a theorem on approximations by trigonometric polynomials holds, too.

Let us also add, roughly speaking, that a bridge between almost automorphic and $S^{p}$-a.p. functions create Stepanov-like almost automorphic functions which have been investigated recently in many papers (see e.g. [10, 15] and others).

https://doi.org/10.5186/aasfm.2017.4250

2010 Mathematics Subject Classification: Primary 42A75, 42A85, 34A30, 26A03, 11A55.

Key words: Almost periodic function in view of the Lebesgue measure, best rational approximation, continued fraction, convolution, kth convergent, limit, linear differential equation, Stepanov almost periodic function. 
Stepanov also introduced the notion of almost periodic function in view of the Lebesgue measure (briefly: $\mu$-a.p. function). He then had not called them almost periodic functions in view of the Lebesgue measure but measurably almost periodic functions. In the case of $\mu$-a.p. functions one considers measurable functions which in contradiction to $S^{p}$-a.p. functions do not have to be locally integrable. Hence the mean value of such functions does not have to exist, however, for such functions a theorem on approximation by generalized trigonometric polynomials holds. Let us indicate that a collection of interesting results on $\mu$-a.p. functions one can find for example in the papers $[18,19]$. Moreover, let us emphasize that $\mu$-a.p. functions possess much more complex nature than $S^{p}$-a.p. functions and therefore, according to our best knowledge, their applications in the theory of evolution equations have not been examined so far.

Non-continuous almost periodic functions in particular have applications in biomathematics, electrical nets and in analysis of neuron nets (see e.g. [12]).

The main goal of this article is to examine $\mu$-a.p. functions with particular emphasis on their behavior under convolution (one of our motivation here is the paper [7]). Our considerations are illustrated by collection of nontrivial examples. These considerations allowed us to prove the main result concerning $\mu$-a.p. solutions to a non-homogeneous linear equation with a $\mu$-a.p. non-homogeneous term. Let us emphasize that the situation under consideration differs from the case when one considers either u.a.p. functions or almost automorphic functions. Moreover, we have examined asymptotic behavior of the classical $\mu$-a.p. function, defined by the formula

$$
x \rightarrow \frac{1}{2+\cos x+\cos (x \sqrt{2})} \quad \text { for } x \in \mathbf{R}
$$

To achieve our goal we have used the theory of continued fractions which is described in many monographs on number theory (see e.g. [13]). Moreover, we provide a general rule of calculation of limits of a certain type.

For completeness, in the next section we collect basic definitions and results which will be used in the sequel. Because that section is quite long (we had to focus in it on $\mu$-a.p. functions, $S^{p}$-a.p. functions and we had to prove a few lemmas concerning continued fractions) we did not focus on B-a.p. functions. The reader could find many details concerning these classical almost periodic functions for example in $[1,9,11,16]$ and in many other monographs on this topic.

\section{Preliminaries}

At the beginning of this section we collect basic definitions and results concerning Stepanov almost periodic functions and almost periodic functions in view of the Lebesgue measure.

Let $\Sigma$ be $\sigma$-algebra of subsets of $\mathbf{R}$ which are measurable in the Lebesgue sense, $\mu$ be the Lebesgue measure on $\Sigma$, and let $\mathcal{X}$ be the space of all $\Sigma$-measurable functions $f: \mathbf{R} \rightarrow \mathbf{R}$. For $f, g \in \mathcal{X}$ we set $f=g \Longleftrightarrow f(x)=g(x)$ for $\mu$-a.e. $x \in \mathbf{R}$. Let $p \geq 1$. As usual, by $L^{p}(\mathbf{R})$ we will denote the set of all functions $\mathbf{R} \rightarrow \mathbf{R}$ measurable in the Lebesgue sense, $p$ th power of absolute value of which is integrable in the Lebesgue sense over $\mathbf{R}$. By $L_{\text {loc }}^{p}(\mathbf{R})$ we will denote the set of all functions $\mathbf{R} \rightarrow \mathbf{R}$ measurable in the Lebesgue sense, $p$ th power of absolute value of which is integrable in the Lebesgue 
sense over every bounded subset of $\mathbf{R}$. For $f, g \in L_{\mathrm{loc}}^{p}(\mathbf{R})$. Let us define

$$
D_{S^{p}}(f, g)=\sup _{u \in \mathbf{R}}\left(\int_{u}^{u+1}|f(t)-g(t)|^{p} d t\right)^{\frac{1}{p}} .
$$

It is easily to check that $D_{S^{p}}$ defines a metric on the set $L_{\mathrm{loc}}^{p}$.

For a function $f: \mathbf{R} \rightarrow \mathbf{R}$ by $f_{\tau}$ for $\tau \in \mathbf{R}$ we denote the function $f_{\tau}: \mathbf{R} \rightarrow \mathbf{R}$, defined by the formula $f_{\tau}(x)=f(x+\tau)$, for $x \in \mathbf{R}$.

Definition 1. [20, 1] A number $\tau \in \mathbf{R}$ is said to be $\left(S^{p}, \varepsilon\right)$-almost period (briefly: $\left(S^{p}, \varepsilon\right)$-a.p.) of a function $f \in L_{\mathrm{loc}}^{p}(\mathbf{R}), p \geq 1$, if $D_{S^{p}}\left(f, f_{\tau}\right) \leq \varepsilon$.

Definition 2. [20, 1] A function $f \in L_{\mathrm{loc}}^{p}(\mathbf{R}), p \geq 1$ is said to be $S^{p}$-almost periodic (briefly: $S^{p}$-a.p.), if for every $\varepsilon>0$ there exists a relatively dense set of its $\left(S^{p}, \varepsilon\right)$-almost periods.

For $\eta>0$ and $f, g \in \mathcal{X}$ we define

$$
D(\eta ; f, g):=\sup _{u \in \mathbf{R}} \mu(\{x \in[u, u+1]:|f(x)-g(x)| \geq \eta\}) .
$$

Moreover, we denote $D(\eta ; f):=D(\eta ; f, 0)$.

Now we recall the notion of $(\varepsilon, \eta)$-almost period of a function $f \in \mathcal{X}$ and the definition of an almost periodic function in view of the Lebesgue measure.

Definition 3. [18] Let $f \in \mathcal{X}$. If for $\varepsilon, \eta>0$ we have $D\left(\eta ; f_{\tau}, f\right) \leq \varepsilon$, then the real number $\tau$ is said to be $(\varepsilon, \eta)$-almost period (briefly: $(\varepsilon, \eta)$-a.p.) of the function $f$.

By $E\{\varepsilon, \eta ; f\}$ we will denote the set of all $(\varepsilon, \eta)$-a.p. of the function $f$, that is

$$
E\{\varepsilon, \eta ; f\}:=\left\{\tau \in \mathbf{R}: \sup _{u \in \mathbf{R}} \mu(\{x \in[u, u+1]:|f(x+\tau)-f(x)| \geq \eta\}) \leq \varepsilon\right\} .
$$

Definition 4. [18] A function $f \in \mathcal{X}$ is said to be almost periodic in view of the Lebesgue measure $\mu$ (briefly: $\mu$-a.p.), if for arbitrary numbers $\varepsilon, \eta>0$, the set $E\{\varepsilon, \eta ; f\}$ is relatively dense. By $\widetilde{M}$ we will denote the set of all $\mu$-a.p. functions.

One can define almost periodic functions in view of the Lebesgue measure in the following equivalent way.

Definition 5. [2] Fix $d>0$. A function $f \in \mathcal{X}$ is said to be $\mu$-a.p. or measurably almost periodic, if for any $\varepsilon>0$, the set

$$
E_{d}\{\varepsilon ; f\}:=\{\tau \in \mathbf{R}: \forall u \in \mathbf{R} \quad \mu(\{x \in[u, u+d]:|f(x+\tau)-f(x)| \geq \varepsilon\})<\varepsilon d\}
$$

is relatively dense.

A straightforward reasoning confirms that the above two definitions are equivalent. It is well-known that if $f$ is a bounded $\mu$-a.p. function, then $f$ is $S^{p}$-a.p. for every $p \geq 1$. Moreover, every bounded and uniformly continuous $\mu$-a.p. function is Bohr almost periodic (see [20]). The following results provides nontrivial examples of $\mu$-a.p. functions.

Theorem 1. [18] Let $F: \Omega \rightarrow \mathbf{C}$, where $\Omega=\{x+i y \in \mathbf{C}:-a<y<a\}, a>0$, be a bounded holomorphic function. Assume that the function $g: \mathbf{R} \rightarrow \mathbf{R}$ given by the formula $g(x)=F(x)$ for $x \in \mathbf{R}$ is Bohr almost periodic. Then the function $f$ 
defined by the formula

$$
f(x)= \begin{cases}\frac{1}{g(x)} & \text { for } x \in \mathbf{R} \text { such that } g(x) \neq 0, \\ 0 & \text { for } x \in \mathbf{R} \text { such that } g(x)=0\end{cases}
$$

is $\mu$-a.p.

We are going also to use Theorem 1 in Section 5 (Example 7). Now we are going to describe briefly the notion of the so-called D-convergence.

Definition 6. [18] A sequence $\left(f_{n}\right)$, where $f_{n} \in \mathcal{X}$ for $N \in \mathbf{N}$ is said to be D-convergent to a function $f \in \mathcal{X}$, if the following condition is satisfied

$$
\forall \varepsilon>0 \quad \forall \eta>0 \quad \exists N \in \mathbf{N} \quad \forall n>N \quad D\left(\eta ; f_{n}, f\right)<\varepsilon .
$$

The function $f$ is said to be D-limit of the sequence $\left(f_{n}\right)$.

It can be proved that $\mathrm{D}$-convergence is weaker that the convergence in view of the Lebesgue measure but it is stronger than the local convergence in view of the Lebesgue measure. Moreover, D-convergent is metrizable.

Definition 7. [18] The functional $|\cdot|: \mathcal{X} \rightarrow \mathbf{R}_{+}$is defined by the formula

$$
\mathbf{|} f \mathbf{|}=\sup _{u \in \mathbf{R}} \int_{u}^{u+1} \frac{|f(t)|}{1+|f(t)|} d t, \quad \text { where } f \in \mathcal{X} .
$$

Using the above functional one can define the metric on $\mathcal{X}$ in a classical way. Moreover, one can prove (see [18]) that a sequence $\left(f_{n}\right)$, where $f_{n} \in \mathcal{X}$ for $n \in \mathbf{N}$, is D-convergent to a function $f \in \mathcal{X}$ if and only if $\left(f_{n}\right)$ is convergent to $f$ in view of the metric generated by that functional. The following result describes the important property of a D-limit.

Theorem 2. [18] If a sequence $\left(f_{n}\right)$ of elements of the metric space $\widetilde{M}$ is $D$ convergent to a function $f \in \mathcal{X}$, then $f \in \widetilde{M}$.

In the next definition an important subclass of the space $\mathcal{X}$ is described.

Definition 8. [20] Let $\left(\lambda_{n}\right)$ be an arbitrary sequence of positive numbers, convergent to zero. Define

$$
\widetilde{\mathcal{X}}=\left\{f \in \mathcal{X}: \sup _{u \in \mathbf{R}} \mu\left(\left\{x \in[u, u+1]: \lambda_{n}|f(x)| \geq 1\right\}\right) \rightarrow 0 \quad \text { as } \quad n \rightarrow \infty\right\},
$$

or equivalently

$$
\tilde{\mathcal{X}}=\left\{f \in \mathcal{X}: D\left(1 ; \lambda_{n} f\right) \rightarrow 0 \quad \text { as } n \rightarrow \infty\right\} .
$$

One can check that the above class does not depend on a choice of the sequence $\left(\lambda_{n}\right)$. In what follows we will apply the following

Theorem 3. [18] If $f$ is $\mu$-a.p., then $f \in \widetilde{\mathcal{X}}$.

Now, we are going to collect basic definitions and facts concerning continued fractions which will be needed in the sequel. As usual, every infinite sequence of real numbers $\left\langle a_{0} ; a_{1}, a_{2}, \ldots\right\rangle$ will be called an infinite continued fraction, provided $a_{j} \geq 1$ $(j=1,2, \ldots)$. The numbers $a_{1}, a_{2}, \ldots$ are said to be quotients of the continued fraction, while the number $r_{k}=\left[a_{0} ; a_{1}, \ldots, a_{k}\right]$ is said to be its $k$ th convergent. If all the quotients are positive integers and $a_{0}$ is an integer, then such a continued fraction is said to be an arithmetic continued fraction. One can easily prove that the limit $\lim _{n \rightarrow \infty}\left[a_{0} ; a_{1} \ldots, a_{n}\right]$ exists; it is said to be the value of the continued fraction $\left\langle a_{0} ; a_{1}, a_{2}, \ldots\right\rangle$ and it is denoted by $\left[a_{0} ; a_{1}, a_{2}, \ldots\right]$. For example, it is easy to check 
that $\sqrt{2}-1=[0 ; 2,2, \ldots]$; we will use this equality in the next section. It is easy to establish the following

Lemma 1. If $a_{0} \in \mathbf{R}, a_{1}, \ldots, a_{n}, a_{n}^{\prime} \geq 1$ and $a_{n}^{\prime}>a_{n}$, then

$$
\left|\left[a_{0} ; a_{1}, \ldots, a_{n-1}, a_{n}\right]-\left[a_{0} ; a_{1}, \ldots, a_{n-1}, a_{n}^{\prime}\right]\right| \leq \frac{1}{n} .
$$

For better calculation of convergents of continued fractions we recall the definition of two sequences of polynomials $\left(P_{n}\right)_{n=-1}^{\infty}$ and $\left(Q_{n}\right)_{n=-1}^{\infty}$ which coefficients are nonnegative integers.

Definition 9. Set

$$
P_{-1}=1, \quad Q_{-1}=0, \quad P_{0}\left(x_{0}\right)=x_{0}, \quad Q_{0}\left(x_{0}\right)=1,
$$

and

$$
P_{k+1}\left(x_{0}, \ldots, x_{k+1}\right)=x_{k+1} P_{k}\left(x_{0}, \ldots, x_{k}\right)+P_{k-1}\left(x_{0}, \ldots, x_{k-1}\right),
$$

and

$$
Q_{k+1}\left(x_{0}, \ldots, x_{k+1}\right)=x_{k+1} Q_{k}\left(x_{0}, \ldots, x_{k}\right)+Q_{k-1}\left(x_{0}, \ldots, x_{k-1}\right)
$$

for $k \in \mathbf{N}_{0}$.

Lemma 2. [13] If $a$ is the value of an infinite continued fraction $\left\langle a_{0} ; a_{1}, a_{2}, \ldots\right\rangle$, and $r_{n}=\frac{P_{n}}{Q_{n}}$ is its $n$th convergent, then

$$
\frac{1}{2 Q_{n} Q_{n+1}}<\left|a-\frac{P_{n}}{Q_{n}}\right|<\frac{1}{Q_{n} Q_{n+1}}<\frac{1}{Q_{n}^{2}} .
$$

If the continued fraction $\left\langle a_{0} ; a_{1}, a_{2}, \ldots\right\rangle$ is arithmetic, then the fraction $\frac{P_{n}}{Q_{n}}$ is irreducible.

Definition 10. [13] We say that a rational number $\frac{a}{b}(b>0 ; a$ and $b$ are coprime) is the best rational approximation of a real number $\alpha$, if for all the fractions $\frac{a^{\prime}}{b^{\prime}}$ with positive integers denominators of which are less than $b$, the following inequality holds

$$
|b \alpha-a|<\left|b^{\prime} \alpha-a^{\prime}\right| \text {. }
$$

Theorem 4. [13] Let $\left\langle a_{0} ; a_{1}, a_{2}, \ldots\right\rangle$ be an arithmetic infinite continued fraction of irrational value $\alpha$. Then every rational number, being the best rational approximation of $\alpha$, is equal to some convergent of this fraction and, conversely, for $k \geq 1$, $k$ th convergent of this fraction is the best rational approximation of the number $\alpha$.

Now, we are going to establish a few simple lemmas.

Lemma 3. Let be given an infinite arithmetic continued fraction

$$
\left\langle a_{0} ; a_{1}, a_{2}, \ldots\right\rangle \text {. }
$$

Let us define two sequences $\left(Q_{m}\right)_{m=-1}^{\infty},\left(P_{m}\right)_{m=-1}^{\infty}$ as in Definition 9. Then:

(i) there exists no index $m \in \mathbf{N}$ such that $Q_{m}$ and $Q_{m+1}$ are even numbers;

(ii) there exists no index $m \in \mathbf{N}$ such that $P_{m}$ and $P_{m+1}$ are even numbers.

Proof. Suppose that $Q_{m}$ i $Q_{m+1}$ are even numbers. Let $s$ be the lowest index such that $Q_{s}$ and $Q_{s+1}$ are even numbers. Obviously $1 \leq s \leq m$. We have

$$
Q_{s+1}=a_{s+1} Q_{s}+Q_{s-1},
$$

and therefore

$$
Q_{s-1}=Q_{s+1}-a_{s+1} Q_{s}
$$


We know that $Q_{s}$ i $Q_{s+1}$ are even, so $Q_{s-1}$ is also an even number, which contradicts to the definition of $s$. The proof of (ii) is analogous.

Lemma 4. Let be given an arithmetic infinite continued fraction $\left\langle a_{0} ; a_{1}, a_{2}, \ldots\right\rangle$. If there exists an index $k \in \mathbf{N}$ such that for $n \geq k$ the numbers $a_{n}$ are odd, then in the sequence $\left(\frac{P_{m}}{Q_{m}}\right)_{m=1}^{\infty}$ there are infinitely many fractions with odd numerators as well as odd denominators.

Proof. Suppose that only finitely many terms of the sequence $\left(\frac{P_{m}}{Q_{m}}\right)_{m=1}^{\infty}$ are fractions with odd numerators as well as odd denominators. Hence there exists an index $N \in \mathbf{N}$ such that for every $m \geq N, P_{m}$ or $Q_{m}$ is an even number. Let $M=\max [N, k]$. Then for every $m \geq M, a_{m}$ is an odd number and $P_{m}$ is an even number, or $Q_{m}$ is an even number. It is known that for $m \geq-1, P_{m}$ and $Q_{m}$ are coprime. Two cases may appear. First, suppose that $P_{M}$ is an even number. Then, by Lemma $3, P_{M+1}$ is an odd number. Moreover, $Q_{M}$ is an odd number, so $Q_{M+1}$ is an even number. Because

$$
Q_{M+2}=a_{M+2} Q_{M+1}+Q_{M}
$$

and

$$
P_{M+2}=a_{M+2} P_{M+1}+P_{M},
$$

so $P_{M+2}$ and $Q_{M+2}$ are odd numbers, what gives a contradiction.

Assume now that $P_{M}$ is an odd number. Then $Q_{M}$ is an even number, so $Q_{M+1}$ is an odd number and $P_{M+1}$ is an even number. In view of (1) and (2), we infer that $P_{M+2}$ i $Q_{M+2}$ are odd numbers, what gives a contradiction.

The following lemma is an elementary observation.

Lemma 5. Let $x \in \mathbf{R}$. Then there exists at most one number $n \in \mathbf{Z}$ such that

$$
|x-2 n|<1 \text {. }
$$

Lemma 6. If for some positive integers $k, n$ it holds

$$
|\alpha(2 k+1)-2 n+1|<1
$$

where $\alpha \in \mathbf{R} \backslash \mathbf{Q}$ and $\frac{2 p-1}{2 k+1}$ is $m$ th convergent $(m \geq 1)$ of the number $\alpha$, then $p=n$.

Proof. Since $\frac{2 p-1}{2 k+1}$ is $m$ th convergent of the number $\alpha$, we have

$$
\left|\alpha-\frac{P_{m}}{Q_{m}}\right|<\frac{1}{Q_{m} Q_{m+1}},
$$

and thus

$$
|\alpha(2 k+1)-(2 p-1)|=\left|\alpha Q_{m}-P_{m}\right|<\frac{1}{Q_{m+1}} \leq 1 .
$$

In view of Lemma 5 , the number $n$ is uniquely determined. Hence $p=n$.

Finally, for $\tau>0$ let us define the function $f^{\tau}: \mathbf{R} \rightarrow \mathbf{R}$ by the formula

$$
f^{\tau}(x)=\left|x+\frac{1}{2} \tau-n \tau\right|,
$$

where $n \in \mathbf{Z}$ satisfies the inequalities

$$
(n-1) \tau \leq x<n \tau .
$$

Let us notice that in view of the Archimedes principle, there exists a unique integer $n$ satisfying the inequalities (4), and therefore the function $f^{\tau}$ is correctly defined. 
The following lemma is also a very simple consequence of the Archimedes principle.

Lemma 7. Let $\tau>0$. Then $f^{\tau}(x) \leq \frac{1}{2} \tau$, for every $x \in \mathbf{R}$.

\section{Asymptotic behavior of the classical $\mu$-a.p. function}

In this section we are going to investigate asymptotic behavior of the classical $\mu$-a.p. function given by the formula

$$
f(x)=\frac{1}{2+\cos x+\cos (x \sqrt{2})} \quad \text { for } x \in \mathbf{R} .
$$

More precisely, our goal is to prove the following

Theorem 5. Let the function $f: \mathbf{R} \rightarrow \mathbf{R}$ be defined as in (5). Then for $\lambda<0$ we have $f(x)=o\left(e^{-\lambda x}\right)$ at $+\infty$, that is

$$
\lim _{x \rightarrow+\infty} \frac{e^{\lambda x}}{2+\cos x+\cos (x \sqrt{2})}=0 .
$$

In the proof of Theorem 5 we will use the following convergence test.

Theorem 6. Let $a \in \mathbf{R}$ and let $f, g: \mathbf{R} \rightarrow \mathbf{R}$ be functions satisfying on the interval $(a,+\infty)$ the following conditions:

$1^{\circ} f$ is nonincreasing and $f(x) \geq 0$;

$2^{\circ} g$ is continuous $g(x)>0$;

$3^{\circ}$ all the points from the interval $(a,+\infty)$ at which $g$ attains a local minimum can be arrange in a increasing sequence $\left(a_{n}\right)_{n \in \mathbf{N}}$ divergent to $+\infty$.

Then

whenever

$$
\lim _{x \rightarrow+\infty} \frac{f(x)}{g(x)}=0
$$

$$
\lim _{n \rightarrow+\infty} \frac{f\left(a_{n}\right)}{g\left(a_{n+1}\right)}=0
$$

Proof. In view of $2^{\circ}$ and $3^{\circ}$, on every interval $\left[a_{n}, a_{n+1}\right]$, where $n \in \mathbf{N}$, we have

$$
g(x) \geq \min \left\{g\left(a_{n}\right), g\left(a_{n+1}\right)\right\} \quad \text { for } x \in\left[a_{n}, a_{n+1}\right] .
$$

Thus, by $1^{\circ}$, we get

$$
\frac{f(x)}{g(x)} \leq \frac{f\left(a_{n}\right)}{\min \left\{g\left(a_{n}\right), g\left(a_{n+1}\right)\right\}} \quad \text { for } x \in\left[a_{n}, a_{n+1}\right] .
$$

Because for $n \geq 2$ we have

$$
0 \leq \frac{f\left(a_{n}\right)}{g\left(a_{n}\right)} \leq \frac{f\left(a_{n-1}\right)}{g\left(a_{n}\right)} \rightarrow 0 \text { as } n \rightarrow+\infty
$$

and

SO

$$
\frac{f\left(a_{n}\right)}{g\left(a_{n+1}\right)} \rightarrow 0 \text { as } n \rightarrow+\infty
$$

$$
\lim _{n \rightarrow+\infty} \frac{f\left(a_{n}\right)}{\min \left\{g\left(a_{n}\right), g\left(a_{n+1}\right)\right\}}=0 .
$$

Now, since $a_{n} \rightarrow+\infty$ as $n \rightarrow+\infty$, by (7) we get

$$
\lim _{x \rightarrow+\infty} \frac{f(x)}{g(x)}=0 \text {. }
$$


In the proof of Theorem 5 we will also need the following

Proposition 1. Let the function $g: \mathbf{R} \rightarrow \mathbf{R}$ be defined by the formula

$$
g(x)=1+\cos x+\frac{1}{\sqrt{2}}[1+\cos (x \sqrt{2})] \quad \text { for } x \in \mathbf{R} .
$$

Then

(i) if $z>0$ is a critical point of the function $g$, then $g(z) \geq \frac{1}{2 !}\left(\frac{1}{8 z}\right)^{8}-\frac{1}{4 !}\left(\frac{1}{8 z}\right)^{16}$;

(ii) all the points from the interval $(0,+\infty)$, at which the function $g$ attains a local minimum form an increasing sequence $\left(a_{n}\right)_{n \in \mathbf{N}}$, divergent to $+\infty$;

(iii) for the sequence defined at (ii), the following estimation holds: $a_{n+1}-a_{n} \leq 2 \pi$.

Proof. Let us prove (i). We have

$$
g^{\prime}(x)=0 \quad \Longleftrightarrow \quad-\sin x-\sin (x \sqrt{2})=0 \quad \Longleftrightarrow \quad \sin x=\sin (-x \sqrt{2}) .
$$

The last equation is satisfied for

$$
x=-x \sqrt{2}+2 k \pi \quad \text { or } \quad x=\pi-(-x \sqrt{2})-2 k \pi, \quad \text { where } k \in \mathbf{Z} .
$$

Since we are interested in positive solutions to these equations, we may assume that solutions to the first equation form a sequence $\left(x_{k}\right)_{k \in \mathbf{N}}$, where $x_{k}=(\sqrt{2}-1) 2 k \pi$, while solutions to the second equation form a sequence $\left(y_{k}\right)_{k \in \mathbf{N}}$, where $y_{k}=(\sqrt{2}+$ $1)(2 k \pi-\pi)$. We are going to prove the property (i) separately for each of these sequences.

In view of Theorem 4 and Lemma 2 we know that if $\frac{P_{m}}{Q_{m}}$ is $m$ th convergent of the number $\sqrt{2}-1$ for $m \geq 1$ and $\frac{p}{q}$ is a rational number such that $1 \leq q \leq Q_{m}, p \in \mathbf{Z}$, then the following estimation holds

Hence

$$
\frac{1}{2 Q_{m} Q_{m+1}}<\left|(\sqrt{2}-1)-\frac{P_{m}}{Q_{m}}\right| \leq\left|(\sqrt{2}-1)-\frac{p}{q}\right| .
$$

$$
\frac{q \pi}{2 Q_{m} Q_{m+1}}<|(\sqrt{2}-1) q \pi-p \pi| \quad \text { for } 1 \leq q \leq Q_{m}, p \in Z .
$$

Now we are going to estimate $Q_{m}$ for the continued fraction $[0 ; 2,2, \ldots]$ of the number $\sqrt{2}-1$ from the above as well as from the below. We have

$$
Q_{-1}=0, \quad Q_{0}=1, \quad Q_{m+1}=2 Q_{m}+Q_{m-1} \quad \text { for } m \geq 0 .
$$

Using mathematical induction we will check that $2^{m} \leq Q_{m}$, for $m \geq 1$. For $m=1$ we have

$$
Q_{1}=2 Q_{0}+Q_{-1}=2 \cdot 1+0=2 \geq 2^{1} .
$$

Suppose that the inequality under consideration holds for some index $m \geq 1$. Then

$$
Q_{m+1}=2 Q_{m}+Q_{m-1} \geq 2 Q_{m} \geq 2^{m} \cdot 2=2^{m+1} .
$$

In an analogous way we check that $Q_{m} \leq 3^{m}$, for $m \geq 1$. For $m=1$ we have

$$
Q_{1}=2 \leq 3^{1} \text {. }
$$

Now assume that the inequality under consideration holds for some index $m \geq 1$. Then

$$
Q_{m+1}=2 Q_{m}+Q_{m-1} \leq 3 Q_{m} \leq 3 \cdot 3^{m}=3^{m+1},
$$

because $Q_{m-1}<Q_{m}$ for $m \geq 1$. Hence

$$
2^{m} \leq Q_{m} \leq 3^{m} \text { for every } m \geq 1 \text {. }
$$


Thus, by the inequality (8) we get the following estimation

$$
\frac{1}{3^{2 m+2}}<\frac{q \pi}{3^{2 m+2}} \leq \frac{q \pi}{2 \cdot 3^{m} 3^{m+1}} \leq \frac{q \pi}{2 Q_{m} Q_{m+1}}<|(\sqrt{2}-1) q \pi-p \pi|,
$$

so

$$
\frac{1}{3^{2 m+2}}<|(\sqrt{2}-1) q \pi-p \pi|
$$

for fixed $m \geq 1$ and $1 \leq q \leq Q_{m}$. Since $2^{m} \leq Q_{m}$, the inequality (9) even more so holds for $1 \leq q \leq 2^{m} \leq Q_{m}$ and $p \in \mathbf{Z}(m \geq 1)$.

Now we are going to estimate $g\left(x_{k}\right)$. Let us notice that

$$
g\left(x_{k}\right) \geq 1+\cos x_{k}=1-\cos \left(x_{k}+\pi\right)=1-\cos \left(f^{2 \pi}\left(x_{k}\right)\right),
$$

because cosine is an even and $2 \pi$-periodic function and

$$
f^{2 \pi}\left(x_{k}\right)=\left|x_{k}+\pi-2 n_{k} \pi\right|=\left|(\sqrt{2}-1) 2 k \pi+\pi-2 n_{k} \pi\right| .
$$

By the earlier estimations, we infer that if $1 \leq 2 k \leq 2^{m}$ for any indexes $k, m \in \mathbf{N}$, then

We claim that

$$
\frac{1}{3^{2 m+2}}<\left|(\sqrt{2}-1) 2 k \pi+\pi-2 n_{k} \pi\right|=f^{2 \pi}\left(x_{k}\right) .
$$

$$
\frac{1}{3^{2 \log _{2} k+6}}<f^{2 \pi}\left(x_{k}\right) \quad \text { for every } k \in \mathbf{N} .
$$

Indeed, for $k \in \mathbf{N}$ we find $m=m(k)$ such that the following condition is satisfied

$$
2^{m-1}<2 k \leq 2^{m}
$$

Then the following inequality holds

$$
m<\log _{2} k+2
$$

and thus

$$
\frac{1}{3^{2 \log _{2} k+6}}<\frac{1}{3^{2 m+2}}<f^{2 \pi}\left(x_{k}\right)
$$

Now, let $z=x_{k}$ for some index $k \in \mathbf{N}$. Then

$$
k=\frac{z}{(\sqrt{2}-1) 2 \pi}
$$

and therefore $z>k$, and

$$
\frac{1}{3^{2 \log _{2} z+6}}<\frac{1}{3^{2 \log _{2} k+6}}<f^{2 \pi}\left(x_{k}\right)=f^{2 \pi}(z) .
$$

Moreover, we have

$$
\frac{1}{3^{2 \log _{2} z+6}}>\frac{1}{4^{2 \log _{2} z+6}}>\left(\frac{1}{8 z}\right)^{4} .
$$

Hence for $z=x_{k}, k \in \mathbf{N}$, the following estimation holds

$$
f^{2 \pi}(z)>\left(\frac{1}{8 z}\right)^{4} .
$$

Moreover, by Lemma 7 , we know that $0 \leq f^{2 \pi}(z) \leq \pi$. Hence, because cosine is a decreasing function on the interval $[0, \pi]$, so

$$
\cos \left(\frac{1}{8 z}\right)^{4}>\cos \left(f^{2 \pi}(z)\right),
$$


and thus

$$
1-\cos \left(\frac{1}{8 z}\right)^{4}<1-\cos \left(f^{2 \pi}(z)\right)
$$

Since the following inequality

$$
1-\cos x>\frac{x^{2}}{2}-\frac{x^{4}}{24}
$$

holds for every $x \in(0,1]$, we get

$$
g(z) \geq \frac{1}{2 !}\left(\frac{1}{8 z}\right)^{8}-\frac{1}{4 !}\left(\frac{1}{8 z}\right)^{16} \quad \text { for } z=x_{k}, k \in \mathbf{N} .
$$

Now, we are going to prove (i) in the case when $z=y_{k}$ for $k \in \mathbf{N}$. Let us notice that

$$
\begin{aligned}
g\left(y_{k}\right) & =g((\sqrt{2}+1)(2 k \pi-\pi)) \\
& =1+\cos [(\sqrt{2}+1)(2 k \pi-\pi)]+\frac{1}{\sqrt{2}}\{1+\cos [\sqrt{2}(\sqrt{2}+1)(2 k \pi-\pi)]\} \\
& =1+\cos [\sqrt{2}(2 k \pi-\pi)-\pi]+\frac{1}{\sqrt{2}}\{1+\cos [(2+\sqrt{2})(2 k \pi-\pi)]\} \\
& =1-\cos [\sqrt{2}(2 k \pi-\pi)]+\frac{1}{\sqrt{2}}\{1+\cos [\sqrt{2}(2 k \pi-\pi)]\} \\
& =1+\frac{1}{\sqrt{2}}-\left(1-\frac{1}{\sqrt{2}}\right) \cos [\sqrt{2}(2 k \pi-\pi)] \geq \frac{2}{\sqrt{2}}=\sqrt{2} .
\end{aligned}
$$

Therefore $g\left(y_{k}\right) \geq \sqrt{2}$. Hence, if $z=y_{k}$ for some $k \in \mathbf{N}$, then $g(z) \geq \sqrt{2}$ and $z>1$. Then

$$
g(z) \geq \sqrt{2}>\frac{1}{2 \cdot 8^{8}}>\frac{1}{2 \cdot 8^{8}}-\frac{1}{4 ! 8^{16}} \geq \frac{1}{2 !}\left(\frac{1}{8 z}\right)^{8}-\frac{1}{4 !}\left(\frac{1}{8 z}\right)^{16}
$$

because the function $\varphi$, defined by the formula

$$
\varphi(z):=\frac{1}{2 !}\left(\frac{1}{8 z}\right)^{8}-\frac{1}{4 !}\left(\frac{1}{8 z}\right)^{16}
$$

is decreasing on the interval $\left(\frac{1}{8 \sqrt[8]{6}},+\infty\right)$. The proof of (i) is complete.

Now, we are going to prove (ii). By (i) we know that if $z>0$ is a critical point of the function $g$, then either it is a term of the sequence $\left(x_{n}\right)_{n \in \mathbf{N}}$ or a term of the sequence $\left(y_{n}\right)_{n \in \mathbf{N}}$. Now, we establish that all the critical points belonging to the interval $(0,+\infty)$ can be arranged in a increasing sequence $\left(c_{n}\right)_{n \in \mathbf{N}}$ divergent to $+\infty$. The sequence $\left(x_{n}\right)_{n \in \mathbf{N}}$ is an arithmetic one with the common difference of $2 \pi(\sqrt{2}-1)$, while the sequence $\left(y_{n}\right)_{n \in \mathbf{N}}$ is an arithmetic one with the common difference of $2 \pi(\sqrt{2}+1)$. Moreover, for any $m, n \in \mathbf{N}$ we have $x_{n} \neq y_{m}$ and $x_{1}<y_{1}$. Hence between two consecutive terms of the sequence $\left(x_{n}\right)_{n \in \mathbf{N}}$ there can be at most one term of the sequence $\left(y_{n}\right)_{n \in \mathbf{N}}$. Thus let $\left(n_{k}\right)_{k \in \mathbf{N}}$ be a sequence of positive integers such that $x_{n_{k}}<y_{k}<x_{n_{k}+1}$, for every $k \in \mathbf{N}$. The sequence $\left(n_{k}\right)_{n \in \mathbf{N}}$ is strictly 
increasing, obviously. No, we are going to construct the sequence $\left(c_{n}\right)_{n \in \mathbf{N}}$. Let

$$
\begin{aligned}
c_{n} & :=x_{n} \quad \text { for } \quad 1 \leq n \leq n_{1} ; \\
c_{n_{1}+1} & :=y_{1} ; \\
c_{n} & :=x_{n-1} \quad \text { for } \quad n_{1}+2 \leq n \leq n_{2}+1 ; \\
c_{n_{2}+2} & :=y_{2} ; \\
c_{n} & :=x_{n-2} \quad \text { for } \quad n_{2}+3 \leq n \leq n_{3}+2 ; \\
c_{n_{3}+3} & :=y_{3}
\end{aligned}
$$

and, in general,

$$
\begin{aligned}
c_{n} & :=x_{n} \quad \text { for } \quad 1 \leq n \leq n_{1} ; \\
c_{n} & :=x_{n-k} \text { for } n_{k}+k+1 \leq n \leq n_{k+1}+k, \quad \text { where } k \in \mathbf{N} \\
c_{n_{k}+k} & :=y_{k} \quad \text { for } k \in \mathbf{N} .
\end{aligned}
$$

Then $\left(c_{n}\right)_{n \in \mathbf{N}}$ is an increasing sequence containing all the positive critical points of the function $g$.

The function $g$ attains local extreme values at all the critical points, because

$$
g^{\prime \prime}(x)=-\cos x-\sqrt{2} \cos (x \sqrt{2})
$$

and

$$
g^{\prime \prime}\left(x_{k}\right)=(-1-\sqrt{2}) \cos (2 \sqrt{2} k \pi), \quad g^{\prime \prime}\left(y_{k}\right)=(1-\sqrt{2}) \cos [\sqrt{2}(2 k \pi-\pi)] .
$$

Since

$$
2 \sqrt{2} k \pi \neq \frac{\pi}{2}+2 s \pi \quad \text { and } \quad \sqrt{2}(2 k \pi-\pi) \neq \frac{\pi}{2}+2 r \pi \quad \text { for } s, r \in \mathbf{Z} \text {, }
$$

we have $g^{\prime \prime}\left(x_{n}\right) \neq 0$ and $g^{\prime \prime}\left(y_{m}\right) \neq 0$ for $m, n \in \mathbf{N}$. Hence every two terms of the sequence $\left(c_{n}\right)_{n \in \mathbf{N}}$ either are pairs of type (maximum, minimum) or pairs of type (minimum, maximum). Thus $\left(c_{n}\right)_{n \in \mathbf{N}}$ is a sequence at terms of which the function $g$ attains local maximum values and local minimum values, alternately. Hence there exists a subsequence $\left(a_{n}\right)_{n \in \mathbf{N}}$ of the sequence $\left(c_{n}\right)_{n \in \mathbf{N}}$ such that the function $g$ attains local minimum values at all of its terms.

Now, we prove the property (iii). If $a_{n}=x_{k}$ for some indexes $n, k \in \mathbf{N}$ and in the interval $\left[x_{k}, x_{k+2}\right]$ there is a term of the sequence $\left(y_{m}\right)_{m \in \mathbf{N}}$, then we have the following situation

$$
a_{n}=x_{k}<y_{m}<x_{k+1}<x_{k+2} \quad \text { or } \quad a_{n}=x_{k}<x_{k+1}<y_{m}<x_{k+2},
$$

so the function $g$ attains a local minimum value at the point $x_{k+1}$ or $y_{m}$, respectively, that is $a_{n+1}=x_{k+1}$ or $a_{n+1}=y_{m}$. Hence

$$
a_{n+1}-a_{n}=x_{k+1}-x_{k}=2 \pi(\sqrt{2}-1)<2 \pi
$$

or

$$
a_{n+1}-a_{n}=y_{m}-x_{k}<x_{k+2}-x_{k}=4 \pi(\sqrt{2}-1)<2 \pi,
$$

respectively. If $a_{n}=x_{k}$ for some indexes $n, k \in \mathbf{N}$ and in the interval $\left[x_{k}, x_{k+2}\right]$ there is no term of the sequence $\left(y_{m}\right)_{m \in \mathbf{N}}$, then we have the following situation

$$
a_{n}=x_{k}<x_{k+1}<x_{k+2},
$$

so at the point $x_{k+2}$ the function $g$ attains a local minimum value, that is $a_{n+1}=x_{k+2}$, and thus

$$
a_{n+1}-a_{n}=x_{k+2}-x_{k}=4 \pi(\sqrt{2}-1)<2 \pi .
$$


If however $a_{n}=y_{m}$ for some indexes $n, m \in \mathbf{N}$, then there exists an index $k \in \mathbf{N}$ such that

$$
x_{k}<a_{n}=y_{m}<x_{k+1}<x_{k+2} .
$$

Moreover, in the interval $\left[x_{k}, x_{k+2}\right]$ there could be at most one term of the sequence $\left(y_{m}\right)_{m \in \mathbf{N}}$. Hence the function $g$ attains at the point $x_{k+2}$ a local minimum value, that is $a_{n+1}=x_{k+2}$, so

$$
a_{n+1}-a_{n}=x_{k+2}-y_{m} \leq x_{k+2}-x_{k}=(\sqrt{2}-1) 4 \pi<2 \pi .
$$

Thus, in all the cases we have $a_{n+1}-a_{n}<2 \pi$ for $n \in N$.

Now, we are ready to provide

Proof of Theorem 5. Let us notice that for $x \in(0,+\infty)$ the following inequalities are satisfied

$$
0 \leq \frac{e^{\lambda x}}{2+\cos x+\cos (x \sqrt{2})} \leq \frac{e^{\lambda x}}{1+\cos x+\frac{1}{\sqrt{2}}[1+\cos (x \sqrt{2})]} .
$$

We are going to prove that

$$
\lim _{x \rightarrow+\infty} \frac{e^{\lambda x}}{1+\cos x+\frac{1}{\sqrt{2}}[1+\cos (x \sqrt{2})]}=0 .
$$

Taking $f(x)=e^{\lambda x}$ and $g(x)=1+\cos x+\frac{1}{\sqrt{2}}[1+\cos (x \sqrt{2})]$ for $x \in \mathbf{R}$ and applying Theorem 6 and Proposition 1, under the same notation we have

$$
\begin{aligned}
& \frac{e^{\lambda a_{n}}}{1+\cos \left(a_{n+1}\right)+\frac{1}{\sqrt{2}}\left[1+\cos \left(a_{n+1} \sqrt{2}\right)\right]} \\
& \leq \frac{e^{\lambda a_{n}}}{\frac{1}{2 !}\left(\frac{1}{8 a_{n+1}}\right)^{8}-\frac{1}{4 !}\left(\frac{1}{8 a_{n+1}}\right)^{16}} \leq \frac{e^{-2 \pi \lambda} e^{\lambda a_{n+1}}}{\frac{1}{2 !}\left(\frac{1}{8 a_{n+1}}\right)^{8}-\frac{1}{4 !}\left(\frac{1}{8 a_{n+1}}\right)^{16}} .
\end{aligned}
$$

Moreover,

because

$$
\lim _{n \rightarrow \infty} \frac{e^{-2 \pi \lambda} e^{\lambda a_{n+1}}}{\frac{1}{2 !}\left(\frac{1}{8 a_{n+1}}\right)^{8}-\frac{1}{4 !}\left(\frac{1}{8 a_{n+1}}\right)^{16}}=0
$$

and

$$
\frac{e^{-2 \pi \lambda} e^{\lambda a_{n+1}}}{\frac{1}{2 !}\left(\frac{1}{8 a_{n+1}}\right)^{8}-\frac{1}{4 !}\left(\frac{1}{8 a_{n+1}}\right)^{16}}=\frac{e^{-2 \pi \lambda} e^{\lambda a_{n+1}}}{\frac{1}{2}\left(\frac{1}{8 a_{n+1}}\right)^{8}\left[1-\frac{1}{12}\left(\frac{1}{8 a_{n+1}}\right)^{2}\right]}
$$

$$
\frac{e^{-2 \pi \lambda} e^{\lambda a_{n+1}}}{\frac{1}{2}\left(\frac{1}{8 a_{n+1}}\right)^{8}} \rightarrow 0, \quad \text { and also } \quad \frac{1}{1-\frac{1}{12}\left(\frac{1}{8 a_{n+1}}\right)^{2}} \rightarrow 1 \quad \text { as } n \rightarrow+\infty,
$$

since $a_{n+1} \rightarrow+\infty$. Now, using Proposition 1 we get the equality (11). In view of the inequality (10) the proof of Theorem 5 is complete.

The result we are going to establish now gives more information about the nature of the limit (6). The question is the following: what can we say about that limit if we replace in $(6), e^{\lambda x}$ by an arbitrary function $f$, and $\sqrt{2}$ by an arbitrary real number? The answer gives the following

Theorem 7. For every function $f: \mathbf{R} \rightarrow \mathbf{R}_{+}$, every $a \in \mathbf{R}$ and every $\varepsilon>0$

$$
\exists_{\alpha \in \mathbf{R}} \exists_{x_{n} \rightarrow+\infty} \quad|a-\alpha|<\varepsilon \text { and } \lim _{n \rightarrow \infty} \frac{f\left(x_{n}\right)}{2+\cos x_{n}+\cos \left(\alpha x_{n}\right)}=+\infty .
$$


Proof. Let us fix $f: \mathbf{R} \rightarrow \mathbf{R}_{+}, a \in \mathbf{R}$ and $\varepsilon>0$. We may assume that $a \notin$ $\mathbf{Q}$, since the set $\mathbf{R} \backslash \mathbf{Q}$ is dense in $\mathbf{R}$. Now, we are going to construct a number $\alpha$ satisfying the above conditions. That number $\alpha$ will be a value of an infinite arithmetic continued fraction

$$
\left\langle a_{0}, a_{1}, \ldots\right\rangle \text {. }
$$

First, from the representation of $a$ as the infinite continued fraction $a=\left[b_{0} ; b_{1}, \ldots\right]$ we choose $s$ initial terms of the sequence in such a way the condition $\frac{1}{s}<\frac{\varepsilon}{2}$ to be satisfied. Let us put $a_{n}=b_{n}$ for $0 \leq n \leq s$. Further, having $m(m \geq s)$ initial terms of the sequence $\left(a_{n}\right)$, as $a_{m+1}$ we take an arbitrary odd number satisfying the inequality

$$
a_{m+1}>\frac{1}{Q_{m}}\left(\pi \sqrt{\frac{m}{2 f\left(Q_{m} \pi\right)}}-Q_{m-1}\right) .
$$

We can do that, because the values $Q_{m}, Q_{m-1}$ are defined, since we already know $m$ initial terms of the representation of the number $\alpha$. Then, by Lemma 1 we get

$$
|\alpha-a| \leq\left|\alpha-\left[b_{0} ; b_{1}, \ldots, b_{s_{0}}\right]\right|+\left|\left[b_{0} ; b_{1}, \ldots, b_{s_{0}}\right]-a\right|<\varepsilon .
$$

Let $y_{k}=\pi+2 k \pi$ for $k \in \mathbf{N}$. We have

$$
\frac{f\left(y_{k}\right)}{2+\cos y_{k}+\cos \left(\alpha y_{k}\right)}=\frac{f\left(y_{k}\right)}{1+\cos \left(\alpha y_{k}\right)}=\frac{f\left(y_{k}\right)}{1-\cos \left(\alpha y_{k}+\pi\right)}=\frac{f\left(y_{k}\right)}{1-\cos f^{2 \pi}\left(\alpha y_{k}\right)} \text {. }
$$

The following estimation obviously holds on the interval $(0, \pi]$

$$
\frac{2}{x^{2}} \leq \frac{1}{1-\cos x} \text {. }
$$

Thus, because $0<f^{2 \pi}\left(\alpha y_{k}\right) \leq \pi$, we get

$$
\frac{2 f\left(y_{k}\right)}{\left[f^{2 \pi}\left(\alpha y_{k}\right)\right]^{2}} \leq \frac{f\left(y_{k}\right)}{1-\cos f^{2 \pi}\left(\alpha y_{k}\right)}
$$

Since beginning with the index $s$ all the denominators $a_{n}$ are odd, by Lemma 4 in the sequence of the convergents $\left(r_{m}\right)$ there are infinitely many fractions with odd numerators as well as odd denominators. Let $\left(r_{m_{l}}\right)$ be a subsequence of the sequence $\left(r_{m}\right)$ such that $Q_{m_{1}}>1$ and $P_{m_{l}}, Q_{m_{l}}$ are odd numbers. Then for every $l \in \mathbf{N}$ there exists $k_{l} \in \mathbf{N}$ such that

$$
\pi Q_{m_{l}}=y_{k_{l}} .
$$

The condition (12) is satisfied because the sequence $\left(\frac{y_{k}}{\pi}\right)$ actually is the sequence of all odd numbers greater than 1 . Moreover, the sequences $\left(m_{l}\right)$ and $\left(k_{l}\right)$ are strictly increasing sequences of positive integers. We have

$$
f^{2 \pi}\left(\alpha y_{k_{l}}\right)=\left|\alpha Q_{m_{l}} \pi-\left(2 n_{l}-1\right) \pi\right|<\pi .
$$

Because

So

$$
\left|\alpha-\frac{P_{m_{l}}}{Q_{m_{l}}}\right|<\frac{1}{Q_{m_{l}} Q_{m_{l}+1}}
$$

$$
\left|\alpha Q_{m_{l}} \pi-P_{m_{l}} \pi\right|<\frac{\pi}{Q_{m_{l}+1}} \leq \pi .
$$

Hence, because $P_{m_{l}}$ is odd and $n_{l}$ is uniquely determined, so, by the Lemma 6 , $2 n_{l}-1=P_{m_{l}}$. We have

$$
m_{l}<2 f\left(y_{k_{l}}\right)\left(\frac{Q_{m_{l}+1}}{\pi}\right)^{2}
$$


because

Therefore

$$
a_{m_{l}+1}>\frac{1}{Q_{m_{l}}}\left(\pi \sqrt{\frac{m_{l}}{2 f\left(Q_{m_{l}} \pi\right)}}-Q_{m_{l}-1}\right) .
$$

so

$$
a_{m_{l}+1} Q_{m_{l}}+Q_{m_{l}-1}>\pi \sqrt{\frac{m_{l}}{2 f\left(Q_{m_{l}} \pi\right)}},
$$

and thus

$$
Q_{m_{l}+1}^{2}>\pi^{2} \frac{m_{l}}{2 f\left(Q_{m_{l}} \pi\right)}
$$

Hence, we have

$$
2 f\left(y_{k_{l}}\right)\left(\frac{Q_{m_{l}+1}}{\pi}\right)^{2}>m_{l} \text {. }
$$

$m_{l}<2 f\left(y_{k_{l}}\right)\left(\frac{Q_{m_{l}+1}}{\pi}\right)^{2} \leq \frac{2 f\left(y_{k_{l}}\right)}{\left[f^{2 \pi}\left(\alpha y_{k_{l}}\right)\right]^{2}} \leq \frac{f\left(y_{k_{l}}\right)}{1-\cos f^{2 \pi}\left(\alpha y_{k_{l}}\right)}=\frac{f\left(y_{k_{l}}\right)}{2+\cos y_{k_{l}}+\cos \alpha y_{k_{l}}}$.

Therefore

$$
\lim _{l \rightarrow \infty} \frac{f\left(y_{k_{l}}\right)}{2+\cos y_{k_{l}}+\cos \alpha y_{k_{l}}}=+\infty .
$$

We put $x_{l}=y_{k_{l}}$, for $l \in \mathbf{N}$, what completes the proof.

\section{Some comments on Stepanov almost periodic functions}

It is well-known that if $f$ is a bounded $\mu$-a.p. function, then it is $S^{p}$-a.p. function for every $p \geq 1$. Below we establish a necessary and sufficient condition for a $\mu$-a.p. function to be an $S^{1}$-a.p.

Remark 1. Let us notice that if $f$ is a locally integrable function, then applying the absolute continuity of the integral, we get

$$
\forall u \in \mathbf{R} \quad \forall \varepsilon>0 \quad \exists \delta>0 \quad \forall A \subset[u, u+1], \mu(A) \leq \delta \Longrightarrow \int_{A}|f(t)| d t \leq \varepsilon .
$$

In what follows we will be interested in the case when this property is satisfied uniformly in view of $u \in \mathbf{R}$, that is when the following condition holds

(13) $\forall \varepsilon>0 \quad \exists \delta>0 \quad \forall u \in \mathbf{R} \quad \forall A \subset[u, u+1], \mu(A) \leq \delta \Longrightarrow \int_{A}|f(t)| d t \leq \varepsilon$.

The condition (13) implies the local integrability, because it is enough to notice that

$$
\int_{u}^{u+1}|f(t)| d t \leq\left[\frac{1}{\delta}\right]+1
$$

where $\delta>0$ is chosen for $\varepsilon=1$ and $\left[\frac{1}{\delta}\right]$ denotes éntier of $\frac{1}{\delta}$. Moreover, the above inequality implies that

$$
\sup _{u \in \mathbf{R}} \int_{u}^{u+1}|f(t)| d t<+\infty
$$

Remark 2. Let us notice that any bounded function measurable in the Lebesgue sense, satisfies the condition (13), since

$$
\int_{A}|f(t)| d t \leq \int_{A} M d t=\mu(A) M \leq \delta M
$$

Applying absolute continuity of the Lebesgue integral we infer also that in the case of a measurable periodic function it means that the function under consideration is 
locally integrable or, equivalently, if $T>0$ is a period of such a function $f$, then the condition (13) is equivalent to the inequality $\int_{0}^{T}|f(t)| d t<+\infty$.

For $\delta \in(0,1]$ and $f \in L_{\text {loc }}^{1}(\mathbf{R})$ put

$$
\varphi(\delta)=\sup _{u \in \mathbf{R}} \sup _{A \subset[u, u+1], \mu(A) \leq \delta} \int_{A}|f(t)| d t .
$$

Then the condition (13) is equivalent to the equality

$$
\lim _{\delta \rightarrow 0^{+}} \varphi(\delta)=0 \text {. }
$$

The following result can be found in the paper [17]. For convenience of the reader we are going to state it along with the proof.

Theorem 8. A locally integrable $\mu$-a.p. function $f$ is $S^{1}$-a.p. if and only if

$$
\lim _{\delta \rightarrow 0^{+}} \varphi(\delta)=0
$$

Proof. Suppose that (14) is satisfied and fix $\varepsilon>0$. Then there exists $\delta>0$ such that $\varphi(\delta) \leq \frac{\varepsilon}{3}$. Choose $\tau \in E\left\{\delta, \frac{\varepsilon}{3} ; f\right\}$. Then, for $u \in \mathbf{R}$ we have

$$
\begin{aligned}
& \int_{u}^{u+1}|f(t+\tau)-f(t)| d t \\
& \leq \int_{A_{u}}|f(t+\tau)| d t+\int_{A_{u}}|f(t)| d t+\int_{A_{u}^{\prime}}|f(t+\tau)-f(t)| d t \leq \varepsilon
\end{aligned}
$$

where

and

$$
A_{u}=\left\{t \in[u, u+1]:|f(t+\tau)-f(t)| \geq \frac{\varepsilon}{3}\right\}
$$

$$
A_{u}^{\prime}=[u, u+1] \backslash A_{u} .
$$

Therefore $D_{S^{1}}\left(f_{\tau}, f\right) \leq \varepsilon$. Thus each $\left(\delta, \frac{\varepsilon}{3}\right)$-a.p. of $f$ is $\left(S^{1}, \varepsilon\right)$-a.p. of $f$. Since $f$ is $\mu$-a.p., the set $E\left\{\delta, \frac{\varepsilon}{3} ; f\right\}$ is relatively dense what implies that $f$ is $S^{1}$-a.p.

Now, assume that $f$ is $S^{1}$-a.p. and that the condition (13) is not satisfied. Then there exist $\varepsilon>0$ and sets $A_{u_{n}}, n \in \mathbf{N}$, such that $\mu\left(A_{u_{n}}\right) \leq \frac{1}{n}, A_{u_{n}} \subset\left[u_{n}, u_{n+1}\right]$ and $\int_{A_{u_{n}}}|f(t)| d t>\varepsilon$. Since $f$ is $S^{1}$-a.p., from the sequence $\left(u_{n}\right)$ one can extract a subsequence $\left(u_{n_{k}}\right)$ such that $f_{u_{n_{k}}} \rightarrow \tilde{f}$ in the topology defined by the metric $D_{S^{1}}$, where $f_{u_{n}}(x)=f\left(x+u_{n}\right)$. Without loss of a generality let us assume that $f_{u_{n}} \rightarrow \tilde{f}$ in that topology. Let us define $A_{n}=A_{u_{n}}-u_{n}$ for $n \in \mathbf{N}$. Then $A_{n} \subset[0,1]$ and $\int_{A_{n}}\left|f_{u_{n}}(t)\right| d t>\varepsilon$. Moreover, there exists an index $n \in \mathbf{N}$ such that

$$
\int_{0}^{1}\left|f_{u_{n}}(t)-\tilde{f}(t)\right| d t<\frac{\varepsilon}{2} \quad \text { and } \quad \int_{A_{n}}|\tilde{f}(t)| d t<\frac{\varepsilon}{2} .
$$

Then, we have

$$
\begin{aligned}
\frac{\varepsilon}{2} & >\int_{0}^{1}\left|f_{u_{n}}(t)-\tilde{f}(t)\right| d t \geq \int_{A_{n}}\left|f_{u_{n}}(t)-\tilde{f}(t)\right| d t \geq \int_{A_{n}}|| f_{u_{n}}(t)|-| \tilde{f}(t)|| d t \\
& \geq \int_{A_{n}}\left|f_{u_{n}}(t)\right|-|\tilde{f}(t)| d t \geq \varepsilon-\int_{A_{n}}|\tilde{f}(t)| d t>\frac{\varepsilon}{2},
\end{aligned}
$$

what completes the proof. 
Now, we are going to investigate the convolution of $S^{1}$-a.p. functions with the function $g_{\lambda}: \mathbf{R} \rightarrow \mathbf{R}(\lambda<0)$ given by the formula

$$
g_{\lambda}(x)= \begin{cases}e^{\lambda x}, & \text { for } x \geq 0 \\ 0, & \text { for } x<0\end{cases}
$$

Let us notice that in such a case we have

$$
\left(f * g_{\lambda}\right)(x)=\int_{-\infty}^{+\infty} f(t) g_{\lambda}(x-t) d t=\int_{-\infty}^{x} f(t) e^{\lambda(x-t)} d t=e^{\lambda x} \int_{-\infty}^{x} f(t) e^{-\lambda t} d t .
$$

Remark 3. Let us notice that for a locally integrable function the existence of the convolution (for every $x \in \mathbf{R}$ ) of a locally integrable function $f$ with the function $g_{\lambda}$ is equivalent to the condition

$$
\left|\int_{-\infty}^{0} f(t) e^{-\lambda t} d t\right|<+\infty
$$

Moreover, by the above equality and the fact that $f g_{\lambda}$ is also locally integrable, it follows that the convolution under consideration is a continuous function.

The result we are going to prove now is a useful test which allows to check if the convolution of a given $S^{1}$-a.p. with the function $g_{\lambda}$ exists, as well as if it is a $\mu$-a.p. function.

Theorem 9. If a function $\mu$-a.p. $f$ satisfies the condition (13), then the convolution $f * g_{\lambda}$ exists for every $x \in \mathbf{R}$ and it is a uniformly almost periodic function.

Proof. First, we prove that the convolution $f * g_{\lambda}$ exists, so we check that

$$
\left|\int_{-\infty}^{0} f(t) e^{-\lambda t} d t\right|<+\infty .
$$

We have

$$
\begin{aligned}
\left|\int_{-\infty}^{0} f(t) e^{-\lambda t} d t\right| & \leq \int_{-\infty}^{0}|f(t)| e^{-\lambda t} d t=\sum_{-n=0}^{+\infty} \int_{n-1}^{n}|f(t)| e^{-\lambda t} d t \\
& \leq \sum_{-n=0}^{+\infty} e^{-\lambda n} \int_{n-1}^{n}|f(t)| d t<+\infty
\end{aligned}
$$

because $\sup _{u \in \mathbf{R}} \int_{u}^{u+1}|f(t)| d t<+\infty$ and the series $\sum_{-n=0}^{+\infty} e^{-\lambda n}$ is convergent.

We are going to establish now that $f * g_{\lambda}$ is a uniformly almost periodic function. Fix $\varepsilon>0$. Since $f$ satisfies the condition (13), there exists $\delta>0$ such that

$$
\varphi(\delta) \leq \frac{\varepsilon}{3 \sum_{-m=-1}^{+\infty} e^{-\lambda m}}
$$

Moreover, let

$$
\eta^{\prime}=\frac{\varepsilon}{3 \sum_{-m=-1}^{+\infty} e^{-\lambda m}} \quad \text { and } \quad \tau \in E\left\{\delta, \eta^{\prime} ; f\right\}
$$

For $u \in \mathbf{R}$ let us define the following sets

$$
A_{u}=\left\{t \in[u, u+1]:|f(t+\tau)-f(t)| \geq \eta^{\prime}\right\} \quad \text { and } \quad A_{u}^{\prime}=[u, u+1] \backslash A_{u} .
$$


Then for $x \in \mathbf{R}$ we have

$$
\begin{aligned}
\left|\left(f * g_{\lambda}\right)(x+\tau)-\left(f * g_{\lambda}\right)(x)\right| & =\left|e^{\lambda(x+\tau)} \int_{-\infty}^{x+\tau} f(t) e^{-\lambda t} d t-e^{\lambda x} \int_{-\infty}^{x} f(t) e^{-\lambda t} d t\right| \\
& \leq e^{\lambda x} \int_{-\infty}^{x}|f(t+\tau)-f(t)| e^{-\lambda t} d t \\
& \leq e^{\lambda[x]} \int_{-\infty}^{[x]+1}|f(t+\tau)-f(t)| e^{-\lambda t} d t \\
& =e^{\lambda[x]} \sum_{-n=-[x]}^{+\infty} \int_{n}^{n+1}|f(t+\tau)-f(t)| e^{-\lambda t} d t \\
& =e^{\lambda[x]} \sum_{-n=-[x]}^{+\infty} e^{-\lambda(n+1)} \int_{n}^{n+1}|f(t+\tau)-f(t)| d t \\
= & e^{\lambda[x]} \sum_{-n=-[x]}^{+\infty} e^{-\lambda(n+1)}\left(\int_{A_{n+\tau}}|f(t)| d t+\int_{A_{n}}|f(t)| d t\right. \\
& \left.+\int_{A_{n}^{\prime}}|f(t+\tau)-f(t)| d t\right) \leq \varepsilon .
\end{aligned}
$$

It means that $E\left\{\delta, \eta^{\prime} ; f\right\} \subset E\left\{\varepsilon ; f * g_{\lambda}\right\}$. Obviously, if the convolution $f * g_{\lambda}$ exists, then it is a continuous function. Hence $f * g_{\lambda}$ is a uniformly almost periodic function.

The construction which we are going to present in the next example will be used in a few subsequent examples. In this example we will need the following simple

Lemma 8. Let $f:(a, b) \rightarrow[0,+\infty)$ be a nonincreasing function. Moreover, let $U \subset(a, b)$ any set which is measurable in the Lebesgue sense and such that $\mu(U) \leq \delta$, where $0<\delta<b-a$. Then

$$
\int_{U} f(t) d t \leq \int_{(0, \delta)} f(t) d t
$$

Example 1. Let us define the sets

$$
A_{n}=2 \cdot 3^{n} \mathbf{Z}-3^{n} ; \quad n \in \mathbf{N}
$$

and the sequence of functions $\left(f_{n}\right)$ :

$$
f_{n}(x)= \begin{cases}\frac{1}{\sqrt{x-z}}, & \text { for } x \in\left[z+\frac{1}{n+1}, z+\frac{1}{n}\right), z \in A_{n}, \\ 0, & \text { otherwise, }\end{cases}
$$

for $n \in \mathbf{N}$. Every function $f_{n}$ is well defined, since for $z \neq z^{\prime}$, the sets $\left[z+\frac{1}{n+1}, z+\frac{1}{n}\right)$ and $\left[z^{\prime}+\frac{1}{n+1}, z^{\prime}+\frac{1}{n}\right)$ are disjoint. Let

$$
f=\sum_{n=1}^{\infty} f_{n}
$$

The function $f$ is well defined, since for every interval $[z, z+1], z \in \mathbf{Z}$ only finitely many functions $f_{n}$ are not equal zero on this interval. Indeed, let us fix $z \in \mathbf{Z}$. Then there exists an index $n_{0} \in \mathbf{N}$ such that

$$
-3^{n_{0}}<z<z+1<3^{n_{0}} .
$$


Then $z, z+1 \notin A_{n_{0}}$. We have $A_{n+1} \subset A_{n}$ for $n \in \mathbf{N}$, because

$$
2 \cdot 3^{n+1} z-3^{n+1}=2 \cdot 3^{n}(3 z-1)-3^{n},
$$

for $z \in \mathbf{Z}$. Thus $z, z+1 \notin A_{n}$ for $n \geq n_{0}$. Moreover, if for some $n \in \mathbf{N}, z \in A_{n}$, then there exists $m \in \mathbf{N}$ such that $z \in A_{m} \backslash A_{m+1}$ and if $[u, u+1] \cap[z, z+1] \neq \emptyset$ for $u \in \mathbf{R}$ and $z \in A_{n}$, then for $x \in[u, u+1] \backslash[z, z+1]$ we have $f(x)=0$. Now, we establish that the sequence of function $\left(g_{k}\right)$, defined by the formulae

$$
g_{k}=\sum_{n=1}^{k} f_{n}
$$

is D-convergent to the function $f$.

Fix $\varepsilon, \eta>0$. Then for every $z \in \mathbf{Z}$, the functions $f$ and $g_{k}$ are equal on the interval $\left[z+\frac{1}{k+1}, z+1\right)$. For $u \in \mathbf{R} \mathrm{w}$ have

$$
\mu\left(\left\{x \in[u, u+1]: f(x)=g_{k}(x)\right\}\right) \geq 1-\frac{1}{k+1} .
$$

Hence, we get

$$
\sup _{u \in \mathbf{R}} \mu\left(\left\{x \in[u, u+1]:\left|f(x)-g_{k}(x)\right|<\eta\right\}\right) \geq 1-\frac{1}{k+1},
$$

so

$$
\sup _{u \in \mathbf{R}} \mu\left(\left\{x \in[u, u+1]:\left|f(x)-g_{k}(x)\right| \geq \eta\right\}\right) \leq \frac{1}{k+1} .
$$

Hence, for sufficiently large $k$ we have $D\left(\eta ; f, g_{k}\right) \leq \varepsilon$, what means that the sequence $\left(g_{k}\right)$ is D-convergent to the function $f$. All the functions $g_{k}$ are measurable in the Lebesgue sense as well as $2 \cdot 3^{k}$-periodic. Hence $g_{k}$ is $\mu$-a.p. for any $k \in \mathbf{N}$ and $f$ is $\mu$-a.p. as the limit of the D-convergent sequence of $\mu$-a.p. functions.

Now, we are going to prove that $f$ satisfies the condition (13). Fix $\varepsilon>0$. Let us take arbitrary $u \in \mathbf{R}$ and $A \subset[u, u+1]$ such that $\mu(A) \leq \frac{\varepsilon^{2}}{4}$. Then, if $[u, u+1] \cap[z, z+1] \neq \emptyset$ for some $z \in A_{n} \backslash A_{n+1}$, applying Lemma 8, we get

$$
\begin{aligned}
\int_{A} f(t) d t & =\int_{A \cap(z, z+1)} f(t) d t \leq \int_{A \cap(z, z+1)} \frac{1}{\sqrt{t-z}} d t \\
& \leq \int_{\left(z, z+\frac{\varepsilon^{2}}{4}\right)} \frac{1}{\sqrt{t-z}} d t=\int_{\left(0, \frac{\varepsilon^{2}}{4}\right)} \frac{1}{\sqrt{t}} d t=\varepsilon .
\end{aligned}
$$

Next, if $[u, u+1] \cap[z, z+1]=\emptyset$ for every $z \in A_{n}$ and $n \in \mathbf{N}$, then we have

$$
\int_{A} f(t) d t=0
$$

In both cases

$$
\int_{A} f(t) d t \leq \varepsilon
$$

Hence it is clear that the condition (13) holds and thus $f * g_{\lambda}$ is a uniformly almost periodic function.

Remark 4. Let us notice that the conclusion in Theorem 9 is stronger than in Bruno-Pankov's theorem proved in [6] (actually they proved that in this situation $f *$ $g_{\lambda}$ is an $S^{p}$-a.p. function). However, as the following example shows, the convolution of an $S^{1}$-a.p. function with a function integrable in the Lebesgue sense, does not have to be uniformly almost periodic. 
Example 2. Let us reconsider the function $f$ from the previous example. Moreover, let

$$
g(x)= \begin{cases}\frac{1}{\sqrt{-x}}, & \text { for } x \in[-1,0) \\ 0, & \text { for } x \in \mathbf{R} \backslash[0,1),\end{cases}
$$

Obviously $g \in L^{1}(\mathbf{R})$. The convolution of $f$ and $g$ is not uniformly almost periodic, because for $z \in A_{n} \backslash A_{n+1}$ we have

$$
\begin{aligned}
(f * g)(z) & =\int_{-\infty}^{+\infty} f(z-t) g(t) d t=\int_{-1}^{0} f(z-t) g(t) d t=\int_{z}^{z+1} f(t) g(z-t) d t \\
& =\int_{z+\frac{1}{n+1}}^{z+1} f(t) g(z-t) d t=\int_{z+\frac{1}{n+1}}^{z+1} \frac{1}{t-z} d t=\int_{\frac{1}{n+1}}^{1} \frac{1}{t} d t=\ln (n+1),
\end{aligned}
$$

because

$$
\sum_{i=1}^{n}\left[z+\frac{1}{i+1}, z+\frac{1}{i}\right)=\left[z+\frac{1}{n+1}, z+1\right) .
$$

Hence this convolution is not a bounded function and thus it is not uniformly almost periodic. However, by the Bruno-Pankov theorem it is an $S^{1}$-a.p. function.

\section{Convolutions of $\mu$-a.p. functions}

At the beginning of this section we provide some comments concerning the convolution of a $\mu$-a.p. function with a function belonging to the space $L^{1}(\mathbf{R})$. The first example will show that the convolution of a $\mu$-a.p. function with a function integrable in the Lebesgue sense does not have to exists.

Example 3. Let $f$ be a periodic extension of the function

$$
h(x)= \begin{cases}\frac{1}{x}, & \text { for } x \in(0,1] \\ 0, & \text { for } x=0\end{cases}
$$

on the set $\mathbf{R}$. Let $g=\chi_{[0,1]}$ be the characteristic function of the interval $[0,1]$. Then for every $x \in \mathbf{R}$ we have

$$
(f * g)(x)=\int_{-\infty}^{+\infty} f(x-t) g(t) d t=\int_{x-1}^{x} f(t) d t=\int_{0}^{1} \frac{1}{t} d t=+\infty
$$

so the convolution $f * g$ does not exists.

Remark 5. If $f$ is a measurable in the Lebesgue sense period function and $g \in L^{1}(\mathbf{R})$, then $f * g$ is periodic and therefore it is also a $\mu$-a.p. function, if it exists. Indeed, if $T>0$ is a period of the function $f$, then for almost all $x \in \mathbf{R}$ we have

$$
(f * g)(x+T)=\int_{-\infty}^{+\infty} f(x+T-t) g(t) d t=\int_{-\infty}^{+\infty} f(x-t) g(t) d t=(f * g)(x) .
$$

Remark 6. Let us notice that a necessary condition for the existence of the convolution $f * \chi_{[0,1]}$ is local integrability of the function $f$. Indeed, if for some $u \in \mathbf{R}$ there were

$$
\int_{u}^{u+\frac{1}{2}}|f(t)| d t=+\infty
$$

then

$$
\int_{u}^{u+\frac{1}{2}} f^{+}(t) d t=+\infty \quad \text { or } \quad \int_{u}^{u+\frac{1}{2}} f^{-}(t) d t=+\infty
$$


Assume that the first equality is satisfied (in the second case the reasoning is similar). Then for $x \in\left[u+\frac{1}{2}, u+1\right]$ we have

$$
\int_{x-1}^{x} f^{+}(t) d t \geq \int_{u}^{u+\frac{1}{2}} f^{+}(t) d t=+\infty
$$

Therefore

$$
\left(f * \chi_{[0,1]}\right)(x)=\int_{x-1}^{x} f(t) d t
$$

is neither finite or defined for $x \in\left[u+\frac{1}{2}, u+1\right]$. Thus the convolution $f * g_{\lambda}$ does not exists. Therefore, in the rest of the paper we are going to consider only locally integrable $\mu$-a.p. functions.

The next example shows that the existence of the convolution of a $\mu$-a.p. function with a function $g \in L^{1}(\mathbf{R})$ does not have to imply that it is $\mu$-a.p.

Example 4. Let

$$
A_{n}:=2 \cdot 3^{n} \mathbf{Z}-3^{n} ; \quad n \in \mathbf{N}
$$

and let us define the sequence of functions $\left(f_{n}\right)$, where

$$
f_{n}(x)= \begin{cases}\frac{1}{x-z}, & \text { for } x \in\left[z+\frac{1}{n+1}, z+\frac{1}{n}\right), \quad z \in A_{n} \\ 0, & \text { otherwise }\end{cases}
$$

for $n \in \mathbf{N}$. The same arguments as in Example 1 establish that $f$ is $\mu$-a.p. Moreover, $f$ is locally integrable in the Lebesgue sense, because on every interval $[u, u+1], u \in \mathbf{R}$ only finitely many functions $f_{n}$ are not identically equal to zero.

Let $g=\chi_{[0,1]}$. Then the convolution $f * g$ exists for every $x \in \mathbf{R}$, however, it is not a $\mu$-a.p. function. Indeed, for $x \in \mathbf{R}$ we have

$$
(f * g)(x)=\int_{-\infty}^{+\infty} f(x-t) g(t) d t=\int_{0}^{1} f(x-t) d t=\int_{x-1}^{x} f(t) d t .
$$

Let $z \in A_{n} \backslash A_{n+1}$. Then

$$
\int_{z}^{z+\frac{1}{2}} f(t) d t=\int_{z+\frac{1}{n+1}}^{z+\frac{1}{2}} \frac{1}{t-z} d t=\int_{\frac{1}{n+1}}^{\frac{1}{2}} \frac{1}{t} d t=\ln \frac{n+1}{2} .
$$

For $x \in\left[z+\frac{1}{2}, z+1\right]$ we have

$$
(f * g)(x)=\int_{x-1}^{x} f(t) d t \geq \int_{z}^{z+\frac{1}{2}} f(t) d t=\ln \frac{n+1}{2} .
$$

Hence, for every $N>0$ we get

$$
\sup _{z \in \mathbf{Z}} \mu(\{x \in[z, z+1]:(f * g)(x) \geq N\}) \geq \frac{1}{2} .
$$

It is well known that if $w$ is a $\mu$-a.p. function, then it satisfies the following condition

$$
\sup _{u \in \mathbf{R}} \mu(\{x \in[u, u+1]:|w(x)| \geq N\}) \rightarrow 0 \text { as } N \rightarrow \infty,
$$

so $f * g \notin \widetilde{\mathcal{X}}$.

Now, we are going to investigate the convolution of $\mu$-a.p. functions with the function $g_{\lambda}: \mathbf{R} \rightarrow \mathbf{R}(\lambda<0)$. 
Remark 7. Similarly as in the case of the characteristic function of the interval $[0,1]$, it can be proved that a necessary condition for the existence of the convolution of a function $f$ with the function $g_{\lambda}$ is local integrability of the function $f$.

The result below gives a sufficient condition under which, in particular, the convolution of a $\mu$-a.p. function with the function $g_{\lambda}$ is not a $\mu$-a.p. function.

Theorem 10. Let $f$ be a nonnegative locally integrable function. If the convolution $f * g_{\lambda}$ exists and

$$
\sup _{u \in \mathbf{R}} \int_{u}^{u+1} f(t) d t=+\infty
$$

then it is not a $\mu$-a.p. function.

Proof. Since the function $f$ satisfies the condition (16), there exists a sequence $\left(u_{n}\right)$ such that

$$
\int_{u_{n}}^{u_{n}+1} f(t) d t \geq n .
$$

For $x \in[0,1]$ we have

$$
\begin{aligned}
(f * g)\left(u_{n}+1+x\right) & =e^{\lambda\left(u_{n}+1+x\right)} \int_{-\infty}^{u_{n}+1+x} f(t) e^{-\lambda t} d t \geq e^{\lambda\left(u_{n}+2\right)} \int_{-\infty}^{u_{n}+1} f(t) e^{-\lambda t} d t \\
& \geq e^{\lambda\left(u_{n}+2\right)} \int_{u_{n}}^{u_{n}+1} f(t) e^{-\lambda t} d t \geq e^{\lambda\left(u_{n}+2-u_{n}\right)} \int_{u_{n}}^{u_{n}+1} f(t) d t \geq e^{2 \lambda} n .
\end{aligned}
$$

Thus for every $N>0$ we have

$$
\sup _{u \in \mathbf{R}} \mu\left(\left\{x \in[u, u+1]:\left(f * g_{\lambda}\right)(x) \geq N\right\}\right)=1 \nrightarrow 0, \quad \text { as } N \rightarrow+\infty .
$$

Therefore $f * g_{\lambda} \notin \widetilde{\mathcal{X}}$ and hence it is not a $\mu$-a.p. function.

Now, we are going to consider a few nontrivial examples.

Example 5. Let us reconsider the function $\mathrm{f}$ defined in Example 2. We are going to prove now that the convolution $f * g_{\lambda}$ exists. Let us consider $z \in A_{m} \backslash A_{m+1}$. Then

$$
\begin{aligned}
\int_{z}^{z+1} f_{n}(t) e^{-\lambda t} d t & \leq e^{-\lambda(z+1)} \int_{z+\frac{1}{m+1}}^{z+\frac{1}{m}} \frac{1}{t-z} d t=e^{-\lambda(z+1)} \int_{\frac{1}{m+1}}^{\frac{1}{m}} \frac{1}{t} d t \\
& =e^{-\lambda(z+1)} \ln \frac{m+1}{m} \leq e^{-\lambda(z+1)} \ln 2 .
\end{aligned}
$$

From the above inequality we infer that $f$ is locally integrable, because on every interval $[z, z+1], z \in \mathbf{Z}$, only finitely many functions $f_{n}$ are not identically equal zero. Now, we are going to estimate

$$
\int_{-\infty}^{0} f_{n}(t) e^{-\lambda t} d t
$$

The support of every function $f_{n}$ for $n \in \mathbf{N}$ is contained in the set $\bigcup_{z \in A_{n}}[z, z+1]$. Since we are interested in $x \leq 0$, we consider $z \in A_{n}$ such that $z+1 \leq 0$. Such $z$ can be arrange in a sequence $\left(z_{k}\right)$, which terms are defined by the formula $z_{k}=$ 
$2 \cdot 3^{n}(1-k)-3^{n}, k=1,2,3, \ldots$ Then

$$
\begin{aligned}
\int_{-\infty}^{0} f_{n}(t) e^{-\lambda t} d t & =\sum_{k=1}^{+\infty} \int_{z_{k}}^{z_{k}+1} f_{n}(t) e^{-\lambda t} d t \leq \ln 2 \sum_{k=1}^{+\infty} e^{-\lambda\left(z_{k}+1\right)} \\
& =e^{-\lambda} \ln 2 e^{-\lambda 3^{n}} \sum_{k=1}^{+\infty} e^{2 k \lambda 3^{n}}=e^{-\lambda} \ln 2 e^{-\lambda 3^{n}} \frac{e^{2 \lambda 3^{n}}}{1-e^{2 \lambda 3^{n}}} \\
& \leq e^{-\lambda} \ln 2 e^{\lambda 3^{n}} \frac{1}{1-e^{6 \lambda}}
\end{aligned}
$$

In view of Beppo-Levy's theorem, we have

$$
\int_{-\infty}^{0} f(t) e^{-\lambda t} d t=\sum_{n=1}^{+\infty} \int_{-\infty}^{0} f_{n}(t) e^{-\lambda t} d t \leq \frac{e^{-\lambda} \ln 2}{1-e^{6 \lambda}} \sum_{n=1}^{+\infty} e^{\lambda 3^{n}}<+\infty
$$

Hence the convolution $f * g_{\lambda}$ exists for all $x \in \mathbf{R}$. The condition (16) follows from (15). Thus this convolution is not a $\mu$-a.p. function.

In the next example we are going to investigate the convolution of the classical $\mu$-a.p. function defined in (5) with the function $g_{\lambda}$.

Example 6. Let $f$ be the function defined in (5). We prove that $f * g_{\lambda}$ exists. For that we establish that

$$
\int_{-\infty}^{a} \frac{e^{-\lambda t}}{2+\cos t+\cos (\sqrt{2} t)} d t<+\infty
$$

for some $a \in \mathbf{R}$. From the proof of Theorem 5 we know that

$$
\frac{e^{\lambda x}}{2+\cos x+\cos (\sqrt{2} x)} \leq \frac{e^{2 \pi \lambda} e^{-a_{n+1}}}{\frac{1}{2 !}\left(\frac{1}{8 a_{n+1}}\right)^{8}-\frac{1}{4 !}\left(\frac{1}{8 a_{n+1}}\right)^{16}}
$$

for $x \in\left[a_{n}, a_{n+1}\right], n \in \mathbf{N}$. Moreover, by Proposition 1 (iii) we know that $a_{n+1}-a_{n}<$ $2 \pi$. Thus $a_{n+1}<x+2 \pi$ and consequently

$$
\frac{e^{\lambda x}}{2+\cos x+\cos (\sqrt{2} x)} \leq \frac{e^{\lambda x}}{\frac{1}{2 !}\left(\frac{1}{8(x+2 \pi)}\right)^{8}-\frac{1}{4 !}\left(\frac{1}{8(x+2 \pi)}\right)^{16}} \quad \text { for } x \geq a_{1} .
$$

Now, it is enough to notice that the integral

$$
\int_{a_{1}}^{+\infty} \frac{e^{\lambda t}}{\frac{1}{2 !}\left(\frac{1}{8(t+2 \pi)}\right)^{8}-\frac{1}{4 !}\left(\frac{1}{8(t+2 \pi)}\right)^{16}} d t
$$

is convergent. Indeed, because

$$
\frac{1}{2 !}\left(\frac{1}{8(x+2 \pi)}\right)^{8}-\frac{1}{4 !}\left(\frac{1}{8(x+2 \pi)}\right)^{16}=\frac{1}{2}\left(\frac{1}{8(x+2 \pi)}\right)^{8}\left[1-\frac{1}{12}\left(\frac{1}{8(x+2 \pi)}\right)^{8}\right]
$$


SO

$$
\begin{aligned}
\int_{a_{1}}^{+\infty} \frac{e^{\lambda t}}{\frac{1}{2 !}\left(\frac{1}{8(t+2 \pi)}\right)^{8}-\frac{1}{4 !}\left(\frac{1}{8(t+2 \pi)}\right)^{16} d t} & =\int_{a_{1}}^{+\infty} \frac{e^{\lambda t}}{\frac{1}{2}\left(\frac{1}{8(t+2 \pi)}\right)^{8}\left[1-\frac{1}{12}\left(\frac{1}{8(t+2 \pi)}\right)^{8}\right]} d t \\
& \leq \int_{a_{1}}^{+\infty} \frac{\frac{1}{2}\left(\frac{1}{8(t+2 \pi)}\right)^{8}\left[1-\frac{1}{12}\left(\frac{1}{8\left(a_{1}+2 \pi\right)}\right)^{8}\right]}{1-\frac{1}{12}\left(\frac{1}{8\left(a_{1}+2 \pi\right)}\right)^{8}} \int_{a_{1}}^{+\infty} \frac{e^{\lambda t}}{\frac{1}{2}\left(\frac{1}{8(t+2 \pi)}\right)^{8}} d t \\
& =\frac{e^{-2 \lambda \pi}}{1-\frac{1}{12}\left(\frac{1}{8\left(a_{1}+2 \pi\right)}\right)^{8}} \int_{a_{1}+2 \pi}^{+\infty} \frac{e^{\lambda t}\left(\frac{1}{8 t}\right)^{8} d t .}{1}
\end{aligned}
$$

The integral

$$
\int_{a_{1}+2 \pi}^{+\infty} \frac{e^{\lambda t}}{\frac{1}{2}\left(\frac{1}{8 t}\right)^{8}} d t
$$

is convergent and thus the integral

$$
\int_{a_{1}}^{+\infty} \frac{e^{\lambda t}}{2+\cos t+\cos (\sqrt{2} t)} d t
$$

is convergent. Obviously

$$
\int_{a_{1}}^{+\infty} \frac{e^{\lambda t}}{2+\cos t+\cos (\sqrt{2} t)} d t=\int_{-\infty}^{-a_{1}} \frac{e^{-\lambda t}}{2+\cos t+\cos (\sqrt{2} t)} d t
$$

so the convolution $f * g_{\lambda}$ exists for $x \in \mathbf{R}$, because $f$ is locally integrable as a continuous function.

A straightforward reasoning shows that the following estimation holds on the interval $[0,2 \pi]$ :

$$
1+\cos x \leq|x-\pi| \text {. }
$$

Similarly one can check that on the interval $[0,2 \pi / \sqrt{2}]$ the following estimation holds

$$
1+\cos (x \sqrt{2}) \leq|x \sqrt{2}-\pi| .
$$

Let $f_{1}, f_{2}: \mathbf{R} \rightarrow \mathbf{R}$ be functions of a period $2 \pi$ and $\frac{2 \pi}{\sqrt{2}}$, respectively, where $f_{1}(x)=$ $|x-\pi|$ for $x \in[0,2 \pi]$ and $f_{2}(x)=|x \sqrt{2}-\pi|$ for $x \in\left[0, \frac{2 \pi}{\sqrt{2}}\right]$. Then for every $x \in \mathbf{R}$ we have $1+\cos x \leq f_{1}(x)$ and $1+\cos x \sqrt{2} \leq f_{2}(x)$. The function $f_{1}$ is equal to zero on the set $\{\pi+2 a \pi: a \in \mathbf{Z}\}$, while the function $f_{2}$ is equal to zero on the set $\left\{\frac{1}{\sqrt{2}}(\pi+2 b \pi): b \in \mathbf{Z}\right\}$. One can easily check that the set $\left\{a+b \frac{1}{\sqrt{2}}: a, b \in \mathbf{Z}\right\}$ is a dense group in $\mathbf{R}$, therefore the set $\left\{2 a \pi+2 b \pi \frac{1}{\sqrt{2}}: a, b \in \mathbf{Z}\right\}$ is dense in $\mathbf{R}$. Hence for every $\eta>0$ there exist $a, b \in \mathbf{Z}$ such that

$$
0<2 a \pi+2 b \pi \frac{1}{\sqrt{2}}+\frac{\pi}{\sqrt{2}}-\pi<\eta .
$$

Putting $a^{\prime}=-a$, we have

$$
0<2 b \pi \frac{1}{\sqrt{2}}+\frac{\pi}{\sqrt{2}}-2 a^{\prime} \pi-\pi<\eta .
$$


Thus there exists a sequence $\left(a_{n}\right)$ and a sequence of positive numbers $\left(\varepsilon_{n}\right)$ such that $\varepsilon_{n} \rightarrow 0$ as $n \rightarrow+\infty\left(\varepsilon_{n} \leq \pi-1\right), a_{n} \in\{\pi+2 a \pi: a \in \mathbf{Z}\}$ and $a_{n}+\varepsilon_{n} \in$ $\left\{\frac{1}{\sqrt{2}}(\pi+2 b \pi): b \in \mathbf{Z}\right\}$. On the interval $\left[a_{n}+\varepsilon_{n}, a_{n}+\varepsilon_{n}+1\right]$ we have $f_{1}(x)=x-a_{n}$ and $f_{2}(x)=x \sqrt{2}-\sqrt{2}\left(a_{n}+\varepsilon_{n}\right)$. Hence

$$
\begin{aligned}
& \int_{a_{n}+\varepsilon_{n}}^{a_{n}+\varepsilon_{n}+1} \frac{1}{f_{1}(t)+f_{2}(t)} d t=\int_{a_{n}+\varepsilon_{n}}^{a_{n}+\varepsilon_{n}+1} \frac{1}{t-a_{n}+\sqrt{2}\left(t-a_{n}-\varepsilon_{n}\right)} d t \\
& =\int_{\varepsilon_{n}}^{\varepsilon_{n}+1} \frac{1}{y+\sqrt{2}\left(y-\varepsilon_{n}\right)} d y=\frac{1}{1+\sqrt{2}} \int_{\varepsilon_{n}}^{\varepsilon_{n}+1} \frac{1}{y-\frac{\sqrt{2}}{1+\sqrt{2}} \varepsilon_{n}} d y \\
& =\frac{1}{1+\sqrt{2}}\left(\ln \left|\varepsilon_{n}+1-\frac{\sqrt{2}}{1+\sqrt{2}} \varepsilon_{n}\right|-\ln \left|\varepsilon_{n}-\frac{\sqrt{2}}{1+\sqrt{2}} \varepsilon_{n}\right|\right) \rightarrow+\infty \quad \text { as } n \rightarrow \infty
\end{aligned}
$$

Therefore, because

$$
\int_{a_{n}+\varepsilon_{n}}^{a_{n}+\varepsilon_{n}+1} \frac{1}{f_{1}(t)+f_{2}(t)} d t \leq \int_{a_{n}+\varepsilon_{n}}^{a_{n}+\varepsilon_{n}+1} \frac{1}{2+\cos t+\cos (t \sqrt{2})} d t
$$

so the function $f$ satisfies the condition (16). Thus the convolution $f * g_{\lambda}$ is not a $\mu$-a.p. function.

It appears that slightly modifying the function from Example 6 one can get the opposite conclusion.

Example 7. Let the function $f: \mathbf{R} \rightarrow \mathbf{R}$ be defined by the formula

$$
f(x)=\frac{1}{\sqrt[4]{2+\cos x+\cos (x \sqrt{2})}} .
$$

We establish that $f$ is $\mu$-a.p. Let us notice that

$$
g(x)=\sqrt[4]{2+\cos x+\cos (x \sqrt{2})}, \quad \text { for } x \in \mathbf{R}
$$

is uniformly almost periodic as the composition of a B-a.p. function with a uniformly continuous function ( $x \rightarrow \sqrt[4]{x}$ is obviously uniformly continuous on the interval $[0,4])$. The function $g$ satisfies the condition of Theorem 1. Moreover, the function $\sqrt[4]{g}$ satisfies the condition of Theorem 1 , because

$$
\sup _{u \in \mathbf{R}} \mu(\{x \in[u, u+1]:|\sqrt[4]{g(x)}| \leq \alpha\})=\sup _{u \in \mathbf{R}} \mu\left(\left\{x \in[u, u+1]:|g(x)| \leq \alpha^{4}\right\}\right) .
$$

Now, we are going to establish, that the function $f$ satisfies the condition (13). We have the following estimation

$$
1+\cos x \leq 2+\cos x+\cos (x \sqrt{2}) \text { for } \quad x \in \mathbf{R} .
$$

Define

$$
h(x)= \begin{cases}\frac{1}{\sqrt[4]{1+\cos x}}, & \text { for } x \in \mathbf{R} \text { such that } 1+\cos x>0, \\ 0, & \text { for } x \in \mathbf{R} \text { such that } 1+\cos x=0 .\end{cases}
$$

Then

$$
\frac{1}{\sqrt[4]{2+\cos x+\cos (x \sqrt{2})}} \leq h(x)
$$


for almost all $x \in \mathbf{R}$. Moreover, the function $h$ is $2 \pi$-periodic, so to prove that the function $h$ satisfies the condition (13), it is enough to show that

$$
\int_{0}^{2 \pi} h(t) d t<+\infty
$$

Using the estimation

$$
1-\cos x \geq \frac{x^{2}}{2}-\frac{x^{4}}{24}
$$

for $x \in(0,1)$, we have

$$
\begin{aligned}
\int_{0}^{2 \pi} \frac{1}{\sqrt[4]{1+\cos t}} d t & =\int_{-\pi}^{\pi} \frac{1}{\sqrt[4]{1-\cos t}} d t=2 \int_{0}^{\pi} \frac{1}{\sqrt[4]{1-\cos t}} d t \\
& =2 \int_{0}^{1} \frac{1}{\sqrt[4]{1-\cos t}} d t+2 \int_{1}^{\pi} \frac{1}{\sqrt[4]{1-\cos t}} d t \\
& \leq 2 \int_{0}^{1} \frac{1}{\sqrt[4]{\frac{t^{2}}{2}-\frac{t^{4}}{24}}} d t+2 \int_{1}^{\pi} \frac{1}{\sqrt[4]{1-\cos 1}} d t \\
& \leq 2 \frac{1}{\sqrt[4]{\frac{1}{2}-\frac{1}{24}}} \int_{0}^{1} \frac{1}{\sqrt{t}} d t+2 \int_{1}^{\pi} \frac{1}{\sqrt[4]{1-\cos 1}} d t<+\infty
\end{aligned}
$$

Hence the function $h$ satisfies the condition (13). Thus the function $f$ satisfies the condition (13). By Theorem 9, the convolution $f * g_{\lambda}$ is a $\mu$-a.p. function.

At the end of this section we provide the example of a continuous $\mu$-a.p. function the convolution of which with $g_{\lambda}$ does not exist.

Example 8. Let $A_{n}=2 \cdot 3^{n} \mathbf{Z}-3^{n}$ for $n \in \mathbf{N}$. To simplify notation let us put $a_{n}=n^{3} e^{-\lambda 3^{n}}$ for $n \in \mathbf{N}$. Define the sequence of functions

$$
f_{n}(x)= \begin{cases}a_{n} x-a_{n}\left(z+\frac{1}{2}-\frac{1}{n+1}\right) & \text { for } x \in\left[z+\frac{1}{2}-\frac{1}{n+1}, z+\frac{1}{2}\right), z \in A_{n} \\ -a_{n} x+a_{n}\left(z+\frac{1}{2}+\frac{1}{n+1}\right) & \text { for } x \in\left[z+\frac{1}{2}, z+\frac{1}{2}+\frac{1}{n+1}\right), z \in A_{n} \\ 0 & \text { for the other } x \in \mathbf{R}\end{cases}
$$

for $n \in \mathbf{N}$. Moreover, let

$$
f=\sum_{n=1}^{+\infty} f_{n}
$$

A similar reasoning as in Example 1 establishes that $f$ is $\mu$-a.p. Because every function $f_{n}$ is continuous and on every bounded interval only finitely many functions $f_{n}$ are not identically equal to zero, so the function $f$ is continuous. Then $f$ is a continuous $\mu$-a.p. function for which we have

$$
\begin{aligned}
\int_{-\infty}^{0} f(x) e^{-\lambda x} d x & \geq \int_{-3^{n}}^{-3^{n}+1} f(x) e^{-\lambda x} d x \geq \int_{-3^{n}}^{-3^{n}+1} f_{n}(x) e^{-\lambda x} d x \\
& \geq e^{-\lambda\left(-3^{n}+1\right)} \int_{-3^{n}}^{-3^{n}+1} f_{n}(x) d x=\frac{n^{3} e^{-\lambda}}{(n+1)^{2}}
\end{aligned}
$$

because $-3^{n} \in A_{n}$ for $n \in \mathbf{N}$. Thus the convolution $f * g_{\lambda}$ does not exist. 


\section{Final remarks about linear differential equations}

In this section we are going to deal with $\mu$-a.p. solutions to the linear differential equation of the form

$$
y^{\prime}(x)=\lambda y(x)+f(x), \quad x \in \mathbf{R} .
$$

As follows from the lemma below, it does make sense for that to examine the function

$$
y(x)=e^{\lambda x} \int_{-\infty}^{x} f(t) e^{-\lambda t} d t, \quad x \in \mathbf{R} .
$$

Lemma 9. Let us consider the equation (17), where $\lambda<0$ and $f \in C(\mathbf{R})$. If the integral $\int_{-\infty}^{0} f(t) e^{-\lambda t} d t$ is well defined and finite, and $y_{0}$ is $\mu$-a.p. solution to this equation, then

$$
y_{0}(x)=e^{\lambda x} \int_{-\infty}^{x} f(t) e^{-\lambda t} d t .
$$

Proof. Because all of the solutions to the above equations are of the shape

$$
y(x)=c e^{\lambda x}+e^{\lambda x} \int_{0}^{x} f(t) e^{-\lambda t} d t,
$$

so there exists $c_{0} \in \mathbf{R}$ such that

$$
y_{0}(x)=c_{0} e^{\lambda x}+e^{\lambda x} \int_{0}^{x} f(t) e^{-\lambda t} d t .
$$

Then, since $y_{0}$ is $\mu$-a.p. solution to the equation under consideration, there exists a sequence $\left(\tau_{n}\right)$ such that $\tau_{n} \rightarrow-\infty$, as $n \rightarrow+\infty$ and a sequence $\left(x_{n}\right)$ such that $x_{n} \in[0,1]$ for $n \in \mathbf{N}$ and the following inequality holds

$$
\left|c_{0} e^{\lambda x_{n}} e^{\lambda \tau_{n}}+e^{\lambda\left(x_{n}+\tau_{n}\right)} \int_{0}^{x_{n}+\tau_{n}} f(t) e^{-\lambda t} d t-c_{0} e^{\lambda x_{n}}-e^{\lambda x_{n}} \int_{0}^{x_{n}} f(t) e^{-\lambda t} d t\right|<1 .
$$

Therefore

$$
\left|c_{0} e^{\lambda \tau_{n}}+e^{\lambda \tau_{n}} \int_{0}^{x_{n}+\tau_{n}} f(t) e^{-\lambda t} d t\right|<e^{-\lambda}+\left|c_{0}\right|+\left|\int_{0}^{x_{n}} f(t) e^{-\lambda t} d t\right| \leq M
$$

for some constant $M>0$. Hence

$$
\left|c_{0}+\int_{0}^{x_{n}+\tau_{n}} f(t) e^{-\lambda t} d t\right|<M e^{-\lambda \tau_{n}} \rightarrow 0 \quad \text { as } n \rightarrow+\infty,
$$

and thus

$$
c_{0}-\int_{-\infty}^{0} f(t) e^{-\lambda t} d t=0 .
$$

Our considerations included in two previous sections lead to the following result.

Theorem 11. Suppose that $\lambda<0$ and $f: \mathbf{R} \rightarrow \mathbf{R}$ is $\mu$-a.p. and continuous. Then one of the following cases holds:

(i) the function (18) is a $\mu$-a.p. to the equation (17);

(ii) the function (18) is a solution to the equation (17), but it is not $\mu$-a.p. function;

(iii) the function (18) is not defined. 
Proof. The case (i) follows from Theorem 9. Actually, under the assumptions of this theorem, the function (18) is a uniformly almost periodic solution to the equation (17).

The case (ii) follows from Example 6, while the case (iii) follows from Example 8.

Remark 8. Let us add in connection with item (i) of the above theorem that Stepanov-like almost automorphic solutions to more general equations than the equation (17) were investigated for example in the papers [10] and [15].

Acknowledgements. We would like to thank the referee for all his/her comments.

\section{References}

[1] Amerio, L., and G. Prouse: Almost periodic functions and functional equations. - Van Nostrand Reinhold Co., No. 30, 1971.

[2] Andres, J., A. M. Bersani, and R.F. Grande: Hierarchy of almost-periodic function spaces. - Rend. Mat. Appl. (7) 26, 2006, 121-188.

[3] Bohr, H.: Zur Theorie der fastperiodischen Funktionen, I. Teil Eine Verallgemeinerung der Theorie der Fourierreihen. - Acta Math. 45, 1925, 29-127.

[4] Bohr, H.: Zur Theorie der fastperiodischen Funktionen, II. Teil Zusammenhang der fastperiodischen Functionen mit Funktionen von unendkich vielen Variablen; gleichmassige Approximation durch trigonometrische Summen. - Acta Math. 46, 1925, 101-214.

[5] Bohr, H.: Zur Theorie der fastperiodischen Funktionen, III. Teil Dirichletentwicklung analytischer Functionen. - Acta Math. 47, 1926, 237-281.

[6] Bruno, G., and A. Pankov: On convolution operators in the spaces of almost periodic functions and $L^{p}$ spaces. - Z. Anal. Anwend. (2) 19, 2000, 359-367.

[7] Bugajewski, D., and T. Diagana: Almost automorhpy of the convolution operator and applications to differential and functional differential equations. - Nonlinear Stud. 13:2, 2006, $129-140$.

[8] Bugajewski, D., and G. N'Guerekata: On the topological structure of almost automorphic and assymptotically almost automorphic solutions of differential and integral equations in abstract spaces. - Nonlinear Anal. 59:8, 2004, 1333-1345.

[9] Corduneau, C.: Almost periodic functions. - Chelesa Publishing Company New York, N.Y., New York, 1989.

[10] Fatajou, S., N. V. Minh, G. N'Guerekata, and A. Pankov: Stepanov-like almost automorphic solutions for nonautonomous evolution equations. - Electr. J. Evol. Equ. 121, 2007, $1-11$.

[11] Levitan, B. M., and V. V. Zhikov: Almost periodic functions and differential equations. Cambridge Univ. Press, Cambridge, 1982.

[12] Marzantowicz, W., and J. Signerska: Firing map of an almost periodic input function. Discrete Contin. Dyn. Syst. Ser. S, 2011, 1032-1041.

[13] Narkiewicz, W.: Number theory. - Polish Scientific Publisher, Warsaw, 2003 (in Polish).

[14] N'Guerekata, G.: Almost automorphic and almost periodic functions in abstract spaces. Kluwer Academic, Plenum Publishers, New York, 2001.

[15] N'Guerekata, G., and A. Pankov: Stepanov-like almost automorphic functions and monotone evolution equations. - Nonlinear Anal. 19, 2008, 2658-2667.

[16] PAnkov, A.: Bounded and almost periodic solutions of nonlinear operator differential equations. - Kluwer Academic Publishers Group, Dordrecht, 1990.

[17] Stepanov, W. W.: Über einige Verallgemeinerungen der fastperiodischen Funktionen. - Ann. Math. 95, 1926, 437-498. 
[18] Stoiński, S.: Almost periodic functions in the Lebesgue measure. - Comment. Math. Prace Mat. 34, 1994, 189-198.

[19] Stoiński, S.: On compactness of almost periodic functions in the Lebesgue measure. - Fasc. Math. 30, 1999, 171-175.

[20] Stoiński, S.: Almost periodic functions. - Scientific Publisher AMU, Poznań, 2008 (in Polish).

Received 9 July 2016 • Accepted 13 January 2017 